\title{
Optical molasses
}

\author{
P. D. Lett, W. D. Phillips, S. L. Rolston, C. E. Tanner, R. N. Watts, and C. I. Westbrook
}

\author{
Center for Atomic, Molecular and Optical Physics, National Institute of Standards and Technology, Gaithersburg, \\ Maryland 20899
}

Received April 4, 1989; accepted July 27, 1989

\begin{abstract}
We present a summary of the results of a simple two-level theory of Doppler cooling in optical molasses and contrast it with the recent theories of multilevel, polarization-gradient cooling. The effects of single-photon recoil and of trapping in microscopic optical potential wells are also considered. Experiments are described in which the temperature of sodium atoms released from optical molasses is measured and found to be well below the Dopplercooling limit. Measurements of the temperature dependence on many experimental parameters are found to be in good qualitative agreement with the new theories of polarization-gradient cooling.
\end{abstract}

\section{INTRODUCTION}

\section{A. Laser Cooling}

In 1975 two groups, Hänsch and Schawlow at Stanford University $^{1}$ and Wineland and Dehmelt at the University of Washington, ${ }^{2}$ independently introduced the idea of laser cooling. While the language and points of view of the two groups were different, they were describing the same basic phenomenon: the reduction in kinetic energy of moving atoms by the mechanical action of laser radiation at a frequency near an atomic resonance. In the Hänsch-Schawlow view, this cooling results because the Doppler effect makes the radiation pressure on an atom velocity dependent. In the Wineland-Dehmelt view, the reduction in energy results from a Raman process in which transitions from higher to lower kinetic energy states are more likely than the reverse. The Hänsch-Schawlow view is more natural when one is considering the cooling of free atoms, while the Wineland-Dehmelt view is more appropriate when the atoms are strongly bound in a potential well. The equivalence of the two viewpoints has been detailed by Wineland and Itano. ${ }^{3,4}$ This paper is concerned mainly with cooling free atoms, so we work from the Hänsch-Schawlow viewpoint.

Hänsch and Schawlow envisaged a gas of atoms irradiated from all sides by six laser beams along each of the six Cartesian coordinate directions. With the laser frequency tuned below (to the red of) the atomic resonance, the Doppler shift makes it more likely that atoms absorb light from the laser beams that are propagating most nearly opposite their own motion. This slows all the atoms, reducing the temperature of the gas. For an atom held in a trap, such as an ion trap, it is not necessary to have six beams. With an appropriately chosen laser-propagation direction and a sufficiently asymmetric trap potential, even a single laser beam is sufficient to cool all the translational degrees of freedom. This is because during some portion of the trapped ion's orbital motion, its velocity has a component opposite the laser beam.

This kind of laser cooling is often called Doppler cooling because of the crucial role played by the Doppler effect. Several authors ${ }^{5-7}$ calculated the lowest temperature (the Doppler-cooling limit) attainable through this process in the low-intensity limit and found that the thermal energy was approximately equal to the energy width of the resonant transition used for cooling. Later treatments showed that higher intensities do not yield any lower temperatures., ${ }^{8,9}$ Doppler cooling was first demonstrated for trapped ions in 1978.7,10 Since that time, laser cooling has also been demonstrated for atomic beams, in both longitudinal and transverse directions, and for gases of free, neutral atoms. Laser cooling has permitted a variety of interesting developments, such as high-resolution spectroscopy, quantum jumps, Coulomb crystallization, trapping of neutral atoms, and studies of collisions at ultralow energy. The reader is referred to the many review papers and collections of papers on the subject, including this special journal issue. ${ }^{11-20}$

\section{B. Optical Molasses}

In 1985 an additional insight was obtained about the process of laser cooling. While Hänsch and Schawlow realized that the strong damping of the atomic motion by the red-tuned laser beams resulted in rapid cooling, they did not discuss the effect on the atomic motion once equilibrium is reached. In fact, such laser-cooled atoms can exhibit diffusive motion. ${ }^{21,22}$ The atomic velocity is damped so quickly, and the atom moves such a short distance in a damping time, that the atomic motion is similar to Brownian motion. This results in a quasi-confinement of atoms within the threedimensional, six-beam geometry envisaged by Hänsch and Schawlow. Because of the viscous nature of the confinement, this six-beam arrangement has been called optical molasses. By extension, strong cooling accompanied by diffusive motion in one or two dimensions is also called optical molasses.

Optical molasses was first observed by a group at Bell Laboratories. ${ }^{21}$ The reported confinement time and temperature of the atoms were consistent with a theory based on Doppler cooling. The temperature reported was at that time the lowest ever achieved by laser cooling, and it was seen to be the ultimate realization of the Hänsch-Schawlow cooling idea. Shortly thereafter, additional experiments brought this view into serious question. Measurements in our laboratory ${ }^{23}$ showed that several features of optical molasses were in strong disagreement with the theoretical predictions, and the Bell Labs group ${ }^{24}$ discovered an anoma- 
lously long-lived supermolasses that had no place in the traditional theory.

Finally, our group discovered that the temperature of atoms cooled by optical molasses could actually be much lower than the Doppler-cooling limit, the lowest temperature that was thought to be achievable by laser cooling. ${ }^{25}$ This discovery, and its confirmation, ${ }^{26,27}$ prompted a reexamination, focusing on the multilevel or multistate nature of the real atoms involved in laser cooling. Eventually two groups proposed similar theories based on the idea of nonadiabatic motion of a multilevel atom through the optical polarization gradient in molasses. ${ }^{26-29}$ More-recent experimental results from our laboratory have given qualitative support to the new theories.

\section{Scope of This Paper}

This paper concentrates on the research done in our laboratory on optical molasses, with brief discussions of some of the work of other groups. In Section 2 we present the implications of a view of optical molasses based on the HänschSchawlow picture of laser cooling and the idea of viscous confinement. This classical molasses theory is a simple generalization of the well-known two-level, one-dimensional, low-intensity laser-cooling theory. We also discuss the two-level, arbitrary intensity theories worked out by several authors ${ }^{8,9,30,31}$ and how they differ from the low-intensity two-level theory. In Section 3 we give the results of the new theory of polarization-gradient laser cooling (presented in detail by two other groups in papers in this special journal issue ${ }^{28,29}$ ) and discuss their implications. We also consider the influence of discrete recoil, and we discuss possible effects due to the presence of dipole-force potential wells. In Section 4 we briefly summarize the early experimental results on optical molasses, both in our own laboratory and elsewhere, emphasizing the ways in which these results are in disagreement with the classical theory of molasses. We discuss in some detail more-recent measurements in our laboratory designed to test explicitly the new theories of polarization-gradient laser cooling and compare the results with the predictions of the theory. In Section 5 we discuss aspects of optical molasses that are still not well understood and speculate about the future of this field. We do not provide a complete review of the work of other groups studying optical molasses. Rather, we discuss such work as it relates to our own and provide references.

\section{CLASSICAL MOLASSES}

By optical molasses we mean not simply laser cooling in counterpropagating laser beams but also the associated phenomenon of diffusive atomic motion that occurs when the laser cooling produces a sufficiently strong damping force. For the theory of laser cooling, we adopt the viewpoint proposed by Hänsch and Schawlow ${ }^{1}$ and developed in more detail by other authors (see, for example, Refs. 3, 6, 8, and 9) that laser cooling results from the Doppler-effect-induced velocity dependence of the radiation pressure force. This theory, which we call classical molasses, assumes a two-level atom interacting with multiple laser beams, that the laser beams do not interfere with one another, and that the atom does not experience coherent interaction involving successions of absorptions and stimulated emissions. For irradia- tion by a weak laser field in one dimension, this theory gives results identical with the low-intensity limit of less restricted theories of two-level, one-dimensional laser cooling. $8,9,11$ We generalize the simple theory in a straightforward (but approximate) way to include moderate-intensity light fields and two or three dimensions, discussing the utility and validity of the generalizations. Within this classical molasses framework we discuss results such as the Doppler-cooling limit and the diffusive motion of atoms in optical molasses.

\section{A. One-Dimensional Classical Molasses}

\section{Damping Force}

Consider a two-level atom with a frequency interval (ignoring recoil energy) between ground and excited states of $\omega_{0}$, irradiated by a laser beam that we assume to be a plane wave of angular frequency $\omega$ and wavelength $\lambda$. We take the frequency width of the laser to be small compared with all other frequencies in the problem. The detuning of the laser frequency from resonance is given by $\Delta=\omega-\omega_{0}$. An atom moving with velocity $v$ in the direction of propagation of the laser sees the laser frequency Doppler shifted down by $2 \pi v / \lambda$ $=k v$, for a total detuning of $\Delta-k v$. The excited-state population decays radiatively to the ground state at a rate $\Gamma$; the strength of the laser-induced coupling between the ground and excited states is characterized by a saturation intensity $I_{0}$ such that when the laser intensity $I=I_{0}$ the transition is power broadened by a factor of $\sqrt{2}$. The relation between the intensity and the Rabi frequency $\omega_{1}$ is $I / I_{0}=$ $2 \omega_{1}^{2} / \Gamma^{2}$. For light with a wavelength $\lambda$, the momentum carried by one photon is $h / \lambda=\hbar k$. The average force on an atom moving in the positive direction is this momentum times the average rate of absorbing photons:

$$
F_{ \pm}= \pm \hbar k \frac{\Gamma}{2} \frac{I / I_{0}}{1+I / I_{0}+[2(\Delta \mp k v) / \Gamma]^{2}} .
$$

Here the upper (lower) sign refers to the force from a traveling plane wave propagating in the positive (negative) direction. This force, often called the radiation-pressure force, scattering force, or spontaneous force, is in the direction of propagation of the light. Note that the maximum value of this force is $\hbar k \Gamma / 2$ for $I / I_{0} \gg 1$. For sodium atoms irradiated by light at the $D 2$ line, $\lambda=589 \mathrm{~nm}$, a transition for which $\Gamma /$ $2 \pi=10 \mathrm{MHz}$, the acceleration corresponding to this maximum force is $10^{6} \mathrm{~m} / \mathrm{sec}^{2}$. $I_{0}$ for the strongest transition $(F=$ $2, m_{F}=2 \rightarrow F^{\prime}=3, m_{F^{\prime}}=3$ ) is $6 \mathrm{~mW} / \mathrm{cm}^{2}$.

There is no contribution to this average force from the momentum transfer on spontaneous emission because that emission is symmetric. The momentum transfer does, however, cause fluctuations of the force, as we shall see in Subsection 2.A.2.

Now let us consider the case of two counterpropagating laser beams. Assuming that the two waves act independently on the atoms, the average force on the atom is given by $F_{+}$ $+F_{-}$. This assumption is true for a two-level system only in the limit of low intensity: $I / I_{0} \ll 1$. In this case we have

$$
F=\frac{\hbar k \Gamma}{2} \frac{I}{I_{0}} \frac{k v}{\Gamma} \frac{16 \Delta / \Gamma}{1+\frac{8}{\Gamma^{2}}\left(\Delta^{2}+k^{2} v^{2}\right)+\frac{16}{\Gamma^{4}}\left(\Delta^{2}-k^{2} v^{2}\right)^{2}} .
$$

We write the damping force in this way to emphasize that it 


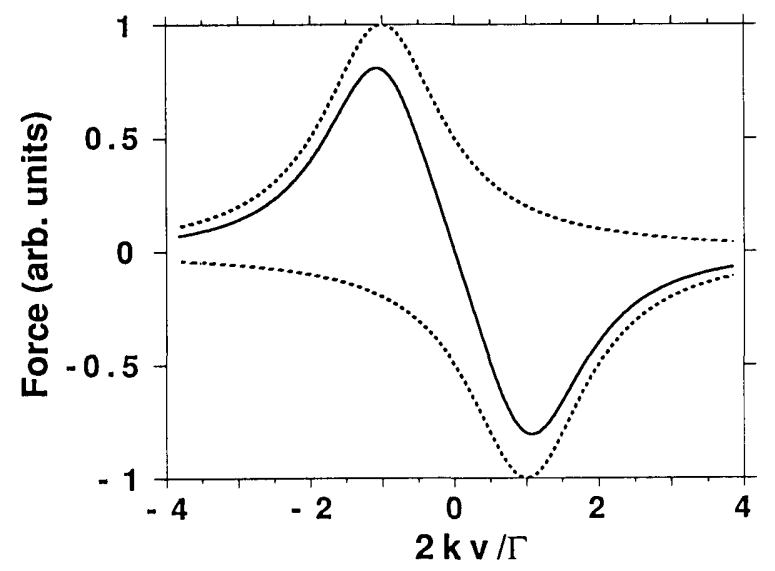

Fig. 1. Force versus velocity for $\Delta=-\Gamma / 2$ and low intensity. The dashed curves are the individual forces due to the two counterpropagating beams, and the solid curve is the net force. Note the linear region around $v=0$.

is the product of the maximum radiation-pressure force, a normalized intensity, the ratio of the Doppler shift to the linewidth, and a factor depending on detuning and velocity. Figure 1 shows $F_{+}, F_{-}$, and their sum for $2 \Delta / \Gamma=-1$ and low intensity.

In the approximation that $|k v| \ll \Gamma$ and $|k v| \ll|\Delta|$ we have

$$
F=4 \hbar k \frac{I}{I_{0}} \frac{k v(2 \Delta / \Gamma)}{\left[1+(2 \Delta / \Gamma)^{2}\right]^{2}} .
$$

For $\Delta<0$ this is a friction force, linear in and opposing $v$. The atoms see the laser beam opposing their motion Doppler shifted closer to resonance, so they absorb photons directed opposite their motion more often than photons directed along their motion. The force is damping for all velocities if $\Delta<0$, and it is linear if $|k v| \ll|\Delta|$ or $|k v| \ll \Gamma$. If $\Delta>0$, the force accelerates the atoms. We assume that $\Delta<0$ in all that follows. In order for Eq. (3) to be meaningful as a damping force, we must further assume that the recoil from a single-photon absorption does not change the atomic velocity so much that the atom is Doppler shifted by a significant fraction of the transition linewidth. That is, $k v_{R} \ll \Gamma$, where $v_{R}=\hbar k / M$ is the velocity change experienced by an atom on the absorption or emission of a single photon. For sodium this recoil is $3 \mathrm{~cm} / \mathrm{sec}$ and $k v_{R} / 2 \pi=50 \mathrm{kHz}$, so $k v_{R} \ll$ $\Gamma$ is satisfied.

Writing this damping force as $F=-\alpha v$, we have the damping coefficient

$$
\alpha=-4 \hbar k^{2} \frac{I}{I_{0}} \frac{(2 \Delta / \Gamma)}{\left[1+(2 \Delta / \Gamma)^{2}\right]^{2}} .
$$

The maximum damping (with $I / I_{0} \ll 1$ and $|k v| \ll|\Delta|, \Gamma$ ) is obtained at $2 \Delta / \Gamma=-1 / \sqrt{3}$, although as we see below this is not the detuning for lowest temperature.

\section{Doppler-Cooling Limit}

The theory of the cooling limit given here is similar in spirit to those given in the usual treatments of laser cooling (see, for example, Refs. $3,8,9$, and 11). The damping force $F=$ $-\alpha v$ leads to a rate of losing kinetic energy of

$$
(\mathrm{d} E / \mathrm{d} t)_{\mathrm{cool}}=F v=-\alpha v^{2} .
$$

This cooling rate is proportional to the kinetic energy, leading to an energy-loss time constant

$$
\tau_{\text {cool }}=-\frac{E}{(\mathrm{~d} E / \mathrm{d} t)_{\text {cool }}}=M / 2 \alpha
$$

and a velocity-damping time constant

$$
\tau_{\text {damp }}=-\frac{v}{(\mathrm{~d} v / \mathrm{d} t)_{\mathrm{cool}}}=M / \alpha .
$$

Now let us consider the rate of increase in energy that is due to heating. The damping force reduces the average velocity of the atoms to zero, but, while the mean velocity becomes zero, the mean squared velocity does not. At the same time that the kinetic energy is being removed by the damping force, it is being supplied by heating from the random nature of the absorption and emission of photons. The damping force that we have derived is only the average force; the fluctuations of the force produce heating. An atom with zero mean velocity is equally likely to absorb a photon from the positive or negative traveling waves. As a result, each absorption represents a step of size $\hbar k$ in a random walk of the momentum of the atom, with equal probability of positive and negative steps. In the same way, each spontaneous emission represents a random-walk step. In a truly one-dimensional problem the spontaneous emissions are along either the positive or negative direction, so that each cycle of absorption followed by spontaneous emission represents two random-walk steps. In a more realistic situation (such as one-dimensional transverse cooling of an atomic beam) in which the spontaneous emissions are into all directions, the average number of steps in the direction of interest (along the laser-propagation axis) is reduced. An isotropic radiation pattern would produce an average of $4 / 3$ steps per absorption-emission, while a dipole pattern would yield $7 / 5$ steps, compared with 2 steps for the truly onedimensional problem. After a given number of steps, the mean square momentum of the atom grows by the number of steps times the square of the photon momentum, $\hbar^{2} k^{2}$. If we denote the total photon scattering rate by $R$, then for the truly one-dimensional case we have

$$
\mathrm{d}\left(p^{2}\right) / \mathrm{d} t=2 \hbar^{2} k^{2} R=2 D_{p},
$$

where $\mathscr{D}_{p}$ is the momentum diffusion constant. Thus the kinetic energy $p^{2} / 2 M$ increases at a rate

$$
(\mathrm{d} E / \mathrm{d} t)_{\text {heat }}=\hbar^{2} k^{2} R / M=\mathcal{D}_{p} / M .
$$

The total scattering rate, in our approximation, is the sum of the scattering rates from the positive and negative traveling waves, which is just the sum of the magnitudes of the individual average forces from these beams divided by the photon momentum $\hbar k$. With the usual approximation that $|k v|$ $\ll|\Delta|$ and $\left.\right|_{\kappa v} \mid \ll \Gamma$ (and that $I / I_{0} \ll 1$ ), we have

$$
(\mathrm{d} E / \mathrm{d} t)_{\text {heat }}=\frac{\hbar^{2} k^{2}}{M} \Gamma \frac{I / I_{0}}{1+(2 \Delta / \Gamma)^{2}} .
$$

Note that the cooling rate, Eq. (5), depends on the square of the velocity, whereas the heating rate, Eq. (10), depends only on the intensity and the detuning.

At equilibrium, the heating and cooling rates are equal. We set $(\mathrm{d} E / \mathrm{d} t)_{\text {heat }}+(\mathrm{d} E / \mathrm{d} t)_{\text {cool }}=0$ and use Eqs. (4), (5), and (10) to obtain a condition on $v^{2}$ : 


$$
v^{2}=\frac{\hbar \Gamma}{4 M} \frac{1+(2 \Delta / \Gamma)^{2}}{2|\Delta| / \Gamma} .
$$

Since the expressions for the heating and cooling rates are time or ensemble average rates, we interpret Eq. (11) as giving the mean square velocity of a group of atoms undergoing laser cooling, or the time average of the squared velocity of a single atom. Taking the thermal energy to be $k_{B} T / 2$ per degree of freedom, $k_{B} T / 2=M v_{\mathrm{rms}}^{2} / 2$, we have in this onedimensional example

$$
k_{B} T=\frac{\mathscr{D}_{p}}{\alpha}=\frac{\hbar \Gamma}{4} \frac{1+(2 \Delta / \Gamma)^{2}}{2|\Delta| / \Gamma} .
$$

This temperature has a minimum value when $2 \Delta / \Gamma=-1$, giving

$$
k_{B} T_{\min }=\hbar \Gamma / 2 .
$$

This is the so called Doppler-cooling limit. For sodium it is equal to $240 \mu \mathrm{K}$, which corresponds to a rms velocity of 30 $\mathrm{cm} / \mathrm{sec}$ in one dimension. This result justifies the assumption that $|k v| \ll \Gamma$, which allowed us to write a damping force linear in velocity. Note also that the recoil velocity $v_{R} \ll$ $v_{\mathrm{rms}}$, which further justifies the treatment of the velocity as a continuous variable for the case of sodium.

This Doppler-cooling limit is equivalent to the statement that the minimum kinetic energy is essentially the same as the energy width of the cooling transition. This is not the only natural energy unit that one might guess as being the cooling limit. One such natural energy, suggested as the cooling limit in Ref. 1, is the kinetic energy where the atomic velocity gives a Doppler shift $k v$ equal to the half linewidth of the cooling transition $\Gamma: k_{B} T_{\text {Dop width }}=M(\Gamma / 2 k)^{2}$. Another, suggested in Ref. 32, is the recoil energy imparted to an atom, initially at rest, when it absorbs the momentum $\hbar k$ of a single photon: $k_{B} T_{R}=\hbar^{2} k^{2} / M$. The actual Dopplercooling limit, Eq. (13), is the geometric mean of $k_{B} T_{R}$ and $k_{B} T_{\text {Dop width. }}$

One may ask whether a laser-cooled atom or collection of atoms can be said to have a temperature. Normally one considers a system with a temperature to be in equilibrium with a reservoir, and in this case the identity of that reservoir is not clear. Even if there are many atoms, they do not interact with one another but only with the radiation field. The radiation field, including the vacuum, may in some sense be a reservoir, but the sense in which this may be true has not been made rigorous. Nevertheless, it can be shown ${ }^{33}$ that the solution of the Fokker-Planck equation for a system acted on by a friction force proportional to velocity, as in Eq. (3), and with a random noise input independent of velocity, such as is provided by the random nature of photon absorption and emissions in the small recoil limit, is a MaxwellBoltzmann distribution. Furthermore, our own Monte Carlo simulations of the laser-cooling process for a single atom, in which every emission and absorption is treated as a random event, leads to a Maxwell-Boltzmann distribution, when averaged over time (see Subsection 3.B below). For this reason, we believe that we are justified in saying that even a single Doppler-cooled atom, under the conditions of small recoil velocity and small thermal velocity, has a temperature.

\section{B. Simple Generalizations of Classical Molasses}

The expression given in Eq. (1) for the force of a single plane wave acting on a two-level atom is valid even when $I / I_{0}>1$. When we include two waves, however, we must make the $I / I_{0}$ $\ll 1$ approximation to obtain Eq. (2). Now a rather obvious way to include a nonnegligible $I / I_{0}$ in Eq. (2) would be to include a saturation term in the denominator corresponding to the total intensity from both waves. ${ }^{23}$ (An alternative method is described in a footnote in Ref. 34.) If, in addition, we wish to include the effect of other pairs of beams directed along the orthogonal coordinate axes in a two- or threedimensional situation, we might simply multiply the saturation term by $N$, the number of dimensions along which we choose to cool (similar to a treatment in Ref. 6). Under these assumptions the damping coefficient is

$$
\alpha=-4 \hbar k^{2} \frac{I}{I_{0}} \frac{2 \Delta / \Gamma}{\left[1+2 N I / I_{0}+(2 \Delta / \Gamma)^{2}\right]^{2}} .
$$

If $N=2$ or 3 , and all axes are equivalent, the velocity and force are vectors with $\mathbf{F}=-\alpha \mathbf{v}$.

Now we might as easily have chosen the saturation term to be simply $I / I_{0}$, which would be equivalent to assuming that each wave independently saturates the atom, with no effect from the other $2 N-1$ waves. Of course, neither choice is correct. Consider just the one-dimensional case for a twolevel atom: the counterpropagating waves will presumably have the same polarization (the one needed to drive the twolevel transition), and there will be a standing wave. As we shall see below, this standing wave will have profound effects on the cooling and heating that are not contained in Eq. (14). If we wish to avoid a standing wave by having orthogonal polarizations in the two waves, the two polarizations will drive different transitions, and we require more than two levels. ${ }^{35}$ Nevertheless, we shall find that the simple approach of Eq. (14) leads to results that are approximately correct for moderate intensity.

Differentiating Eq. (14) with respect to $\Delta$ or $I$, we find the conditions for maximum damping:

$$
\begin{array}{ll}
\text { With fixed detuning: } & \frac{I}{I_{0}}=\frac{(2 \Delta / \Gamma)^{2}+1}{2 N}, \\
\text { With fixed intensity: } & \frac{2 \Delta}{\Gamma}=-\left(\frac{1+2 N I / I_{0}}{3}\right)^{1 / 2} .
\end{array}
$$

Note that the optimum detuning of Eq. (15b) is identical to that derived from Eq. (4) if we replace the linewidth $\Gamma$ with the (assumed) power-broadened linewidth $\Gamma\left(1+2 N I / I_{0}\right)^{1 / 2}$. Simultaneously optimizing the damping with respect to both detuning and intensity, we find that

$$
\alpha=\frac{\hbar k^{2}}{4 N} \quad \text { for } 2 \Delta / \Gamma=-1, \quad I / I_{0}=1 / N .
$$

For sodium, the minimum velocity damping time $M / \alpha$ corresponding to this maximum value for $\alpha$ is $13 \mu \mathrm{sec}$ in one dimension and $40 \mu \mathrm{sec}$ in three dimensions.

The total photon-scattering rate from all $2 N$ waves is

$$
R=\frac{\Gamma}{2} \frac{2 N I / I_{0}}{1+2 N I / I_{0}+(2 \Delta / \Gamma)^{2}},
$$

leading to a diffusion constant $\mathscr{D}_{p}=\hbar^{2} k^{2} R$. With the conditions of Eq. (16) this gives a heating rate $D_{p} / M=$ 
$(N / 2) k_{B} \mathrm{~d} T / \mathrm{d} t$, which for sodium is equivalent to $75 \mu \mathrm{K} / \mu \mathrm{sec}$ in one dimension or $25 \mu \mathrm{K} / \mu \mathrm{sec}$ in three dimensions.

Equating the heating and cooling rates at arbitrary $I$ and $\Delta$, we find from $k_{B} T=D_{p} / N \alpha$ that

$$
k_{B} T=\frac{\hbar \Gamma}{4} \frac{1+2 N I / I_{0}+(2 \Delta / \Gamma)^{2}}{2|\Delta| / \Gamma} .
$$

Note that, in the limit of $I / I_{0} \ll 1$, Eq. (18) is identical to Eq. (12): The low-intensity cooling limit in two or three dimensions is the same as in one dimension. If we substitute the maximum damping conditions of Eq. (16) into Eq. (18) we find that $k_{B} T=\hbar \Gamma$. This is twice the minimum temperature of $\hbar \Gamma / 2$ obtained for $I / I_{0} \ll 1$. At the intensity for maximum damping, the heating has increased more than the damping, resulting in the higher temperature.

While the total kinetic energy is larger in higher dimension, the energy per degree of freedom is the same in all dimensions. This is because we have assumed that in one or two dimensions the emitted photons are all emitted in the line or plane under consideration. In an experimentally realizable situation, the one- or two-dimensional temperature will be lower and will depend on the details of the radiation pattern. In three dimensions we assume that the photons are emitted isotropically. If the emission is not isotropic we may have anisotropic temperatures, that is, different values of $\left\langle v_{i}{ }^{2}\right\rangle$. The anisotropy could depend, for example, on the relative polarization of the various light beams used in the cooling.

As noted above, the generalization to nonnegligible intensity and multiple dimensions is not strictly valid. There is, however, a procedure suggested by Dalibard ${ }^{36}$ that makes this generalization exact: If one alternates each of the $2 \mathrm{~N}$ traveling waves so that each is on for $1 / 2 N$ of the time and they are never on simultaneously, there is no standing wave. The atoms will still see the same average force given by Eq. (14) as long as the alternation time is short compared with the damping time $\tau_{\text {damp }}$ but long compared with the radiative decay time $\Gamma^{-1}$. In that case, we obtain Eq. (14), with $I /$ $I_{0}$ being the average intensity in each beam and $2 N I / I_{0}$ the instantaneous intensity. The conditions for optimum damping are then the same as for our simple generalization. ${ }^{36}$

\section{Cooling with a Single Traveling Wave}

Although we are concerned primarily with cooling by pairs of counterpropagating waves, it is interesting to note that cooling by a single traveling wave gives the same equilibrium temperature as the case discussed above. The single-traveling-wave configuration is used to slow and cool an atomic beam and has been treated extensively elsewhere. ${ }^{22,37,38}$ Here we will deal only with the question of the cooling limit.

A central concern in cooling an atomic beam is to prevent the atoms from detuning far out of resonance with the laser as they decelerate. Two main methods are used in practice: chirp cooling, in which the laser frequency is changed to compensate for the changing atomic Doppler shift, and Zeeman-tuned cooling, in which a spatially varying magnetic field compensates for the Doppler shift. Here we shall assume chirp cooling and note that the situation for Zeeman tuning is essentially the same.

We assume that the atomic beam has a velocity distribution centered about a positive velocity $V^{\prime}$ with a small spread of velocities. From Eq. (1), the force on an atom with velocity $V^{\prime}+v$ is

$$
F^{\prime}=-\hbar k \frac{\Gamma}{2} \frac{I / I_{0}}{1+I / I_{0}+\left\{2\left[\Delta^{\prime}+k\left(V^{\prime}+v\right)\right] / \Gamma\right\}^{2}},
$$

where $\Delta^{\prime}$ is the detuning. The group of atoms with a velocity $V^{\prime}$ will experience a deceleration $a=F^{\prime} / M$. We shall transform into a frame decelerating with these atoms. Furthermore, we shall assume that the detuning of the laser is changed at such a rate that the total detuning, including the Doppler shift associated with the moving frame, remains constant. That is, $\Delta^{\prime}(t)=\Delta^{\prime}(0)-k V^{\prime}(t)$, where $V^{\prime}(t)=$ $V^{\prime}(0)+a t$. This ensures that the force and hence the deceleration remain constant. Writing the force in the decelerating frame, taking into account the transformed velocities and the Doppler shift of the laser frequency, we have

$$
F=-M a-\hbar k \frac{\Gamma}{2} \frac{I / I_{0}}{1+I / I_{0}+[2(\Delta+k v) / \Gamma]^{2}},
$$

where $M a$ is the (fictitious) inertial force in the decelerating frame and is just Eq. (19) evaluated at $v=0$. Substituting for $M a$, using the Doppler-shift transformation $\Delta^{\prime}=\Delta-$ $k V^{\prime}$, and taking $|k v| \ll|\Delta|$ and $|k v| \ll \Gamma$, we find the force in the decelerating frame:

$$
F=2 \hbar k \Gamma \frac{I}{I_{0}} \frac{k v(2 \Delta / \Gamma)}{\left[1+I / I_{0}+(2 \Delta / \Gamma)^{2}\right]^{2}} .
$$

This is exactly the force found from Eq. (14) for cooling by two counterpropagating waves $(N=1)$, except that $2 I / I_{0}$ has been replaced with $I / I_{0}$ since there is now only one wave. Using Eq. (21) to get $\alpha$ and the single-beam scattering rate to get $\mathscr{D}_{p}$, we find that the cooling limit in the decelerating frame is equivalent to the one derived for counterpropagating beams, ${ }^{39,40} \mathrm{Eq}$. (18). When we make no approximation in assuming nonnegligible intensity, the temperature in the decelerating frame is

$$
k_{B} T=\frac{\hbar \Gamma}{4} \frac{1+I / I_{0}+(2 \Delta / \Gamma)^{2}}{2|\Delta| / \Gamma},
$$

which is identical to Eq. (18) if we interpret $I / I_{0}$ as the total intensity seen by the atoms. We have implicitly assumed that the deceleration is allowed to continue long enough for equilibrium to be established. Note also that in the decelerating frame we must have $\Delta<0$ in order to cool. This problem is equivalent to opposing a constant external force with radiation pressure from a single traveling wave. When the radiation pressure force and the external force balance, the cooling limit is given by Eq. (22).

\section{Validity of the Generalizations}

The extension of the classical molasses theory to multiple dimensions is valid, in a two-level system, for low intensity. However, as we have noted above, the extension of the theory to nonnegligible intensity is not valid, except in the case when the molasses beams are alternated so that only one beam is on at a time. When multiple beams are present simultaneously, we cannot consider the beams as acting independently once $I / I_{0} \approx 1$. Inclusion of saturation, as in Subsection 2.B, does not properly account for the effects of having two strong waves simultaneously. One of the key 
processes left out of that treatment is the possibility that an atom may absorb a photon from one of the waves and be stimulated to emit into the other one. At high intensity this can be an important process leading to dipole (gradient) forces and to fluctuations of these forces, which contribute additional heating. Dipole forces have been treated in some detail by other authors (see, for example, Refs. 8, 9, 11, and $30)$.

Here we briefly summarize some features of atomic motion in counterpropagating waves with nonnegligible intensity. We consider two cases: a two-level system in which the counterpropagating beams form a standing wave and a multilevel system in which the the counterpropagating beams are orthogonally polarized and do not form a standing wave. We give only the results and indicate the degree to which these more exact treatments differ from the classical treatment given above.

A number of authors have treated the case of atomic motion in an intense standing wave. Minogin and Serimaa ${ }^{31}$ used a continued fraction approach, which yields exact solutions for arbitrary atomic velocity. Gordon and Ashkin ${ }^{8}$ and $\mathrm{Cook}^{9}$ adopted a perturbative approach valid for small velocities. Dalibard ${ }^{36}$ gives a convenient perturbative expression for the force on an atom moving in a standing wave, where the force has been averaged over a wavelength of light. With $F=-\alpha v$, he finds that

$$
\begin{aligned}
\alpha=-\frac{\hbar k^{2}}{(1+4 s)^{3 / 2}}\{ & \frac{4 s(2 \Delta / \Gamma)}{1+(2 \Delta / \Gamma)^{2}}(1+2 s) \\
& \left.-\frac{2 \Delta}{\Gamma}\left[1+6 s+6 s^{2}-(1+4 s)^{3 / 2}\right]\right\}
\end{aligned}
$$

for $|k v| \ll \Gamma$. Here, $s$ is the detuning-dependent saturation parameter for one of the two traveling waves that compose the standing wave:

$$
s=\frac{I / I_{0}}{1+(2 \Delta / \Gamma)^{2}} .
$$

The features of this standing-wave molasses that are so different from those of classical molasses are due mainly to the ability of the atom to interact successively with both of the counterpropagating traveling waves. Dalibard et al. ${ }^{41}$ have considered a multilevel system interacting with orthogonally polarized counterpropagating waves that eliminates the possibility of such successive interaction. Specifically, they considered a $J=0 \rightarrow J=1$ transition irradiated by oppositely directed $\sigma^{+}$and $\sigma^{-}$waves. Such a configuration does not form a standing wave, and, because of the selection rules on transitions between the $m$ levels, absorption from one wave cannot be followed by stimulated emission from the other. For $|k v| \ll \Gamma$ they find a damping coefficient

$$
\alpha=-\hbar k^{2} \frac{2 s}{1+2 s} \frac{2(2 \Delta / \Gamma)}{(2 \Delta / \Gamma)^{2}+\left\{1+1 / 2 s\left[(2 \Delta / \Gamma)^{2}+1\right]\right\}^{2}},
$$

where $s$ is given by Eq. (24) [this differs slightly from Eq. (3.22) of Ref. 41 because of a different definition of $s$ ].

Figure 2 compares the damping coefficient for classical molasses, Eq. (14), standing-wave molasses, Eq. (23), and $\sigma^{+}-\sigma^{-}$molasses, Eq. (25). For $s \ll 1$ or $I / I_{0} \ll 1$, these all reduce to Eq. (4). For moderate powers, $I / I_{0} \leq 1$, the expressions are not much different. For $I / I_{0}>1$ the standing- wave damping becomes significantly different from the classical and $\sigma^{+}-\sigma^{-}$damping. Only at high power does the $\sigma^{+}$ $\sigma^{-}$damping differ markedly from the classical damping.

One of the most remarkable features of standing-wave molasses is that for moderate to large $I / I_{0}$ and detunings larger than needed to maximize $\alpha$, the sign of $\alpha$ reverses, producing acceleration rather than damping. When the detuning is positive rather than negative, the sign reversal leads to strong damping. This phenomenon has been used to produce blue (stimulated) molasses and is discussed extensively in Refs. 30 and 42.

The standing-wave case also differs from classical molasses in the rate at which heating occurs. Successive interactions can lead to high heating rates at high intensity. At low intensity, however, the heating is approximately the same as for classical molasses. For plane waves in the $\sigma^{+}-\sigma^{-}$case, the heating is essentially the same as for classical molasses. Explicit expressions for the momentum diffusion constants are given in Refs. 8, 9, and 36 for the standing-wave case and Ref. 41 for the $\sigma^{+}-\sigma^{-}$case. The temperature for both of these cases is never lower than the Doppler-cooling limit.

In conclusion, for high intensity the exact solutions for atomic motion in counterpropagating waves can differ substantially from the results of classical molasses. For moderate powers $\left(I / I_{0} \leq 1\right)$, the results of classical molasses are in approximate agreement with the more exact treatments. Both the damping and the heating are somewhat higher in the standing-wave case than in classical molasses, but the general behavior is quite similar. Thus, at least for moderate intensity, classical molasses gives an approximate description of a two-level-system in counterpropagating waves. For the $\sigma^{+}-\sigma^{-}, J=0 \rightarrow J=1$ configuration of Ref. 41, for which successive stimulated processes are not possible, classical molasses gives a good approximation even at moderate intensity.

\section{E. Diffusive Motion in Classical Molasses}

We now consider the motion of atoms in optical molasses in more detail. In Subsection 2.B we found that the minimum velocity-damping time for sodium atoms in three-dimensional classical molasses is $40 \mu \mathrm{sec}$. The three-dimensional rms velocity at the Doppler-cooling limit is $50 \mathrm{~cm} / \mathrm{sec}$, so an atom with that average velocity will move only $20 \mu \mathrm{m}$ during a damping time. This short mean free path indicates that the motion will be diffusive if the extent of the molasses is much larger than $20 \mu \mathrm{m}$.

Following the viewpoint adopted by Chu et al., ${ }^{21}$ we treat the motion of atoms in optical molasses as the Brownian motion of particles in a viscous fluid. ${ }^{43}$ The position diffusion constant $\mathscr{D}_{x}$ is given by

$$
D_{x}=\frac{k_{B} T}{\alpha}=\frac{D_{p}}{N \alpha^{2}}
$$

where we have used $k_{B} T=\mathscr{D}_{p} / N \alpha$. After a time $t_{D}$ the mean square diffusion distance in $N$ dimensions is $N$ times the mean square distance along a single axis:

$$
\left\langle r^{2}\right\rangle=N\left\langle x^{2}\right\rangle=2 N t_{D} \mathcal{D}_{x}=\frac{2 t_{D} D_{p}}{\alpha^{2}} .
$$

Substituting the results of Subsection 2.B into Eq. (27), we obtain 

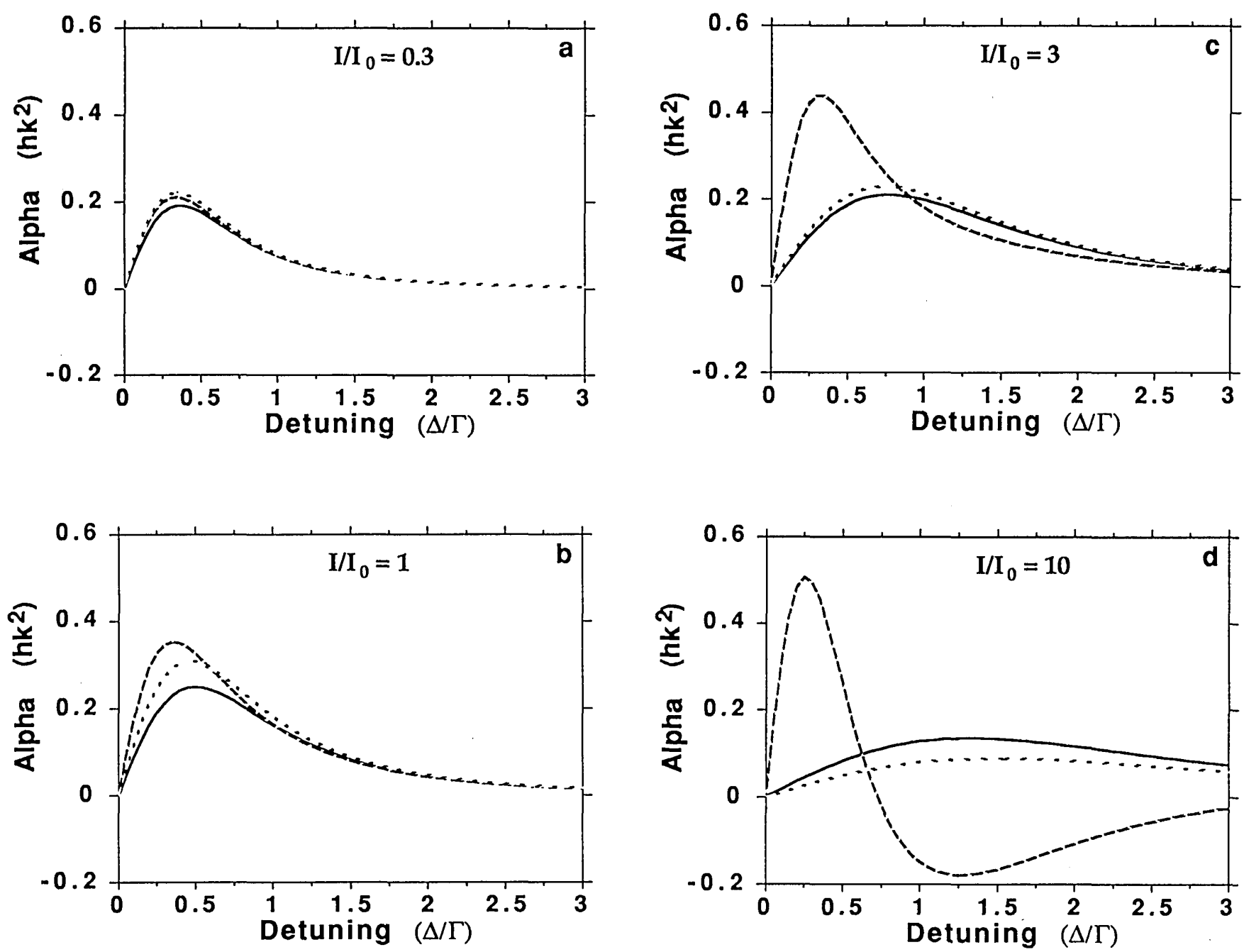

Fig. 2. Damping coefficient $\alpha$ versus detuning red of resonance for classical molasses (solid), standing wave (dashed), and $\sigma^{+}-\sigma^{-}$(dotted), for different intensities. a, $I / I_{0}=0.3 ; \mathrm{b}, I / I_{0}=1.0 ; \mathrm{c}, I / I_{0}=3.0 ; \mathrm{d}, I / I_{0}=10.0$

$$
t_{D}=\frac{8 k^{2}\left\langle r^{2}\right\rangle}{N \Gamma} \frac{I}{I_{0}} \frac{(2 \Delta / \Gamma)^{2}}{\left[1+2 N I / I_{0}+(2 \Delta / \Gamma)^{2}\right]^{3}}
$$

Maximizing the diffusion time with respect to both intensity and detuning, we obtain

$$
t_{D}=\frac{4 k^{2}\left\langle r^{2}\right\rangle}{27 N^{2} \Gamma} \quad \text { for } 2 \Delta / \Gamma=-1, \quad I / I_{0}=1 / 2 N
$$

Note that the condition for maximum diffusion time is slightly different from the condition for maximum damping coefficient given in Eq. (16).

We have assumed that the molasses is of infinite extent. For sodium the time required to diffuse $0.5 \mathrm{~cm}$ in an infinite molasses is $750 \mathrm{msec}$ for the conditions of Eq. (29). In a real situation the molasses region will be formed at the intersection of laser beams of finite extent. Let us assume ${ }^{21}$ that this intersection region is approximated by a sphere of radius $r$ and that any atom reaching the boundary of the sphere is lost. For a variety of initial distributions within the sphere, it can be shown that the number of atoms remaining within the sphere after a time $t$ is a sum of exponentially decaying terms. ${ }^{44}$ The dominant term is

$$
n(t)=n_{0} \exp \left(-t / \tau_{M}\right), \quad \tau_{M}=r^{2} / \pi^{2} \mathscr{D}_{x} .
$$

Using Eqs. (27) and (30) with $N=3$, we also have $\tau_{M}=6 t_{D} /$ $\pi^{2}$. For sodium the maximum exponential time constant for decay of a $0.5-\mathrm{cm}$-radius classical molasses is $450 \mathrm{msec}$.

Now consider the effect of an external force applied to an atom in optical molasses. The atom will acquire a drift velocity such that the damping force cancels the external force:

$$
v_{\text {drift }}=F_{\text {ext }} / \alpha .
$$

If the external force is gravity, and we take optimum damping conditions in three-dimensional sodium molasses, we find that $v_{\text {drift }}=12 \mathrm{Mg} / \hbar k^{2}=0.37 \mathrm{~mm} / \mathrm{sec}$. This is much smaller than the thermal velocity of $50 \mathrm{~cm} / \mathrm{sec}$, and the drift time through a $0.5 \mathrm{~cm}$ radius molasses is $\sim 14 \mathrm{sec}$, which is long compared with the molasses decay time. Therefore we expect little effect from drift due to gravity.

Another, more severe, source of drift is imbalance between the intensities of counterpropagating molasses beams. We assume that in one pair of molasses beams the intensity in one beam is $(1+\epsilon)$ times the intensity in the other beam. 
Generalizing Eq. (1), we have an unbalanced force along the direction of the stronger beam of

$$
F_{\text {im }}=\frac{\hbar k \Gamma \epsilon}{2} \frac{I / I_{0}}{1+2 N I / I_{0}+(2 \Delta / \Gamma)^{2}} .
$$

Using Eq. (14) for $\alpha$, we have

$$
v_{\text {drift }}=\frac{\epsilon \Gamma}{8 k} \frac{1+2 N I / I_{0}+(2 \Delta / \Gamma)^{2}}{2|\Delta| / \Gamma} .
$$

For the optimum damping conditions of Eq. (16) in three dimensions we have $v_{\text {drift }}=\Gamma \epsilon / 2 k$. For sodium $\Gamma / 2 k=3 \mathrm{~m} /$ sec. A $1 \%$ imbalance leads to a $3 \mathrm{~cm} / \mathrm{sec}$ drift velocity, or a drift time for $0.5 \mathrm{~cm}$ of $167 \mathrm{msec}$, considerably shorter than the maximum molasses decay time. Therefore we expect classical molasses to be highly sensitive to imbalance between the opposing beams.

\section{NEW THEORIES OF LASER COOLING}

None of the theories discussed in Section 2 predicts temperatures any lower than the Doppler-cooling limit. In fact, no theoretical treatment of free-atom laser cooling before 1988 of which we are aware predicted a steady-state temperature below that limit. Our laboratory's observation of temperatures well below the Doppler-cooling limit initiated a search for another mechanism for laser cooling.

By mid-1988 two groups, Dalibard and Cohen-Tannoudji at the Ecole Normale Superieure in Paris and Chu and colleagues at Stanford University, had proposed a new mechanism for laser cooling, one that relies in a fundamental way on the degeneracy of the ground state, optical pumping, and the spatial gradient of the polarization of the cooling light. The Ecole Normale group has called this mechanism polarization-gradient laser cooling.

The polarization-gradient damping force is in some ways radically different from the damping force in classical molasses or other earlier theories of laser cooling. The most striking and counterintuitive feature of the new force is that the damping coefficient is independent of laser intensity at low intensity. Since the heating rate is proportional to the intensity, as in classical molasses, this leads to a temperature that decreases linearly as the intensity. At the same time, the range of coolable velocities also decreases with intensity. Recall that for classical molasses the temperature is independent of intensity at low intensity. Furthermore, the magnitude of the new damping coefficient can be considerably higher than the maximum coefficient achievable with classical molasses. As we shall see, this larger damping coefficient results in temperatures well below the classical cooling limit under certain conditions of intensity and detuning.

In Subsection 3.A we briefly summarize the qualitative features of the new theory of multilevel, polarization-gradient laser cooling advanced by the Ecole Normale and Stanford groups. We also quote the major quantitative results for comparison with experiments discussed in Section 4 . For more detail concerning these theories, we refer the reader to the early expositions (Refs. 26 and 27) and especially to the more comprehensive treatments given by these groups in this special journal issue. ${ }^{28,29}$
Besides the polarization-gradient laser-cooling mechanism, other mechanisms have been discussed that are similar in spirit but do not explicitly involve polarization gradients. ${ }^{45}$ These mechanisms involve spatially dependent optical pumping among either degenerate or nondegenerate ground states but will not be discussed further here. All these mechanisms may play some role in a system as complicated as sodium in three-dimensional optical molasses.

In Subsection 3.B we consider the influence of the discreteness of the momentum transfer in laser cooling, an issue of particular significance since the new theories predict minimum kinetic energies comparable with the energy imparted by a single photon recoil. In Subsection 3.C we discuss the possible effect of wavelength-sized dipole-force potential wells on the motion of atoms in optical molasses.

\section{A. Polarization Gradient Cooling}

The Ecole Normale and Stanford groups have considered two separate one-dimensional cases in which the polarization gradient of the light field is responsible for the damping. The first case is counterpropagating waves with orthogonal circular polarization. This $\sigma^{+}-\sigma^{-}$, or corkscrew, configuration produces a local polarization that is linear everywhere with a direction of polarization that rotates a full turn every wavelength. The corkscrew polarization corresponds to a pure rotation of polarization and gives rise to polarizationrotation cooling.

The second case is counterpropagating waves with orthogonal linear polarizations. This linear-perpendicular-linear, or $\pi^{x} \pi^{y}$, configuration has a local polarization that changes from $\sigma^{+}$through elliptical to linear, to elliptical, to $\sigma^{-}$, and back to $\sigma^{+}$in a distance of $\lambda / 2$, but with a period of $\lambda$ when phase is included. This changing character of polarization gives rise to ellipticity gradient cooling.

Polarization-rotation and ellipticity-gradient cooling produce quite different damping coefficients. (See, for example, the plots by Ungar et al..$^{28}$ comparing these damping forces as obtained from numerical solutions of the optical Bloch equations describing the cooling of sodium.) Dalibard and Cohen-Tannoudji ${ }^{29}$ obtain analytic results for the simplest model systems exhibiting the effect and identify distinct physical mechanisms for each case.

In the corkscrew polarization case, for a $J=1 \rightarrow J^{\prime}=2$ optical transition an atom at rest is optically pumped into a distribution of ground $m$ states having alignment (different populations for states with different $|m|$ ) along the polarization axis but no orientation (asymmetry of positive and negative $m$-level population). When the atom moves parallel to the laser-propagation axis, an orientation develops along this axis. The population asymmetry is such that the most populated state is the one that absorbs light most strongly from the circularly polarized wave opposing its motion. This asymmetry in the absorption accounts for the damping force in polarization-rotation cooling. ${ }^{29}$

In the linear-perpendicular-linear case for a $J=1 / 2 \rightarrow J^{\prime}$ $=3 / 2$ optical transition an atom at rest is optically pumped into a distribution of $m$ states that depends on the local, spatially varying, polarization character. The light-shifted energies of these states also vary in space so that a moving atom climbs and descends the hills and valleys of the modulated optical potential. At low velocity the optical pumping 
process always redistributes the $m$-level population such that as much population climbs a hill as descends. For finite velocity, the time lag of the optical pumping process results in more population climbing the hills than descending them, so the velocity is damped. This mechanism is similar to the Sisyphus cooling described in Ref. 42 and is responsible for the ellipticity-gradient cooling. ${ }^{29}$ In both mechanisms, the existence of multiple ground-state sublevels and a long optical pumping time among the sublevels is crucial for obtaining large damping forces and low temperatures.

Ungar et $a l .{ }^{28}$ have treated the same one-dimensional polarizations but for the $J=2 \rightarrow J^{\prime}=3$ case corresponding to the $3 S_{1 / 2}(F=2) \rightarrow 3 P_{3 / 2}\left(F^{\prime}=3\right)$ transition used in laser cooling of sodium. Their numerical solutions of the optical Bloch equations for this more complicated case are in qualitative agreement with the analytic results of Dalibard and Cohen-Tannoudji for the simpler transitions. In particular, for small intensity, and $k v \ll \Gamma$, the theories give a damping force that is independent of laser intensity, linear in the velocity for small velocity, and reaching a maximum value for some critical velocity. Ungar $e t$ al. find that the dependence of the damping on detuning is also in agreement with the analytic results.

For the $\sigma^{+}-\sigma^{-} J=1 \rightarrow J^{\prime}=2$ system Dalibard and CohenTannoudji find [Eq. (5.14) of Ref. 29] a linear damping coefficient

$$
\alpha=-\frac{60}{17} \hbar k^{2} \frac{2 \Delta / \Gamma}{5+(2 \Delta / \Gamma)^{2}} .
$$

This has a maximum value of $\sim 0.8 \hbar k^{2}$ at $\Delta=-\sqrt{5 / 4} \Gamma$. Such a damping coefficient would give a velocity-damping time of $\sim 4 \mu \mathrm{sec}$ in sodium. By comparison, for the $\sigma^{+}-\sigma^{-} J=2 \rightarrow J^{\prime}$ $=3$ system Ungar et al. find numerically a similar detuning dependence, with a maximum $\alpha$ near $\Delta=-\Gamma / 2$, of approximately $4.5 \hbar k^{2}$. It is of particular interest to compare Eq. (34) with Eq. (4) or (14) for classical molasses. At large detuning the classical damping decreases as $1 / \Delta^{3}$, while the polarization-rotation damping decreases only as $1 / \Delta$. Recall also that the maximum classical damping coefficient is $0.25 \hbar k^{2}$ in one dimension.

In the ellipticity-gradient case for $J=1 / 2 \rightarrow J^{\prime}=3 / 2$ Dalibard and Cohen-Tannoudji find [Eq. (4.26) of Ref. 29] that

$$
\alpha=-\frac{3}{2} \hbar k^{2} \frac{2 \Delta}{\Gamma} .
$$

This dependence on detuning is in especially sharp contrast to the case of classical molasses, with the damping actually increasing with detuning. Ungar et al. do not evaluate the detuning dependence for this case, but at a detuning of $\Delta=$ $-2.73 \Gamma$ they find an $\alpha$ of roughly $15 \hbar k^{2}$, whereas for that detuning Eq. (35) would predict $\alpha=8 \hbar k^{2}$ for the lower angular momentum transition. The latter damping coefficient would imply a velocity damping time of $\sim 0.5 \mu \mathrm{sec}$ in sodium.

Dalibard and Cohen-Tannoudji have derived analytic expressions for the momentum diffusion coefficients in the two cases, under conditions of low velocity and intensity. This leads to the temperature as in Section 2 above. For polarization rotation they find [Eqs. (5.18) and (4.37) of Ref. 29] that

$$
k_{B} T=\frac{\hbar \Gamma I / I_{0}}{(2|\Delta| / \Gamma)}\left[\frac{29}{300}+\frac{254}{75} \frac{1}{1+(2 \Delta / \Gamma)^{2}}\right],
$$

whereas for the ellipticity gradient, for $\Delta \gg \Gamma$,

$$
k_{B} T=\frac{\hbar \Gamma I / I_{0}}{8(2|\Delta| / \Gamma)} .
$$

For both cases the temperature is proportional to the intensity and, at large detuning, inversely proportional to detuning. This would seem to imply that arbitrarily low temperatures are possible at sufficiently low intensity or large detuning; however, another feature of the polarization-gradient cooling limits the temperature.

An important result of both the Ecole Normale and Stanford treatments of polarization-gradient cooling is the existence of a critical velocity $v_{c}$ around which the damping force is no longer linear in velocity and above which the damping coefficient decreases. Velocities higher than $v_{c}$ do not experience the strong polarization-gradient damping force implied by Eqs. (34) and (35), so $v_{c}$ represents a capture range for the polarization-gradient force. Velocities much higher than $v_{c}$ will be only slowly damped, mainly by the classical molasses force, whereas those comparable with or lower than $v_{c}$ will be quickly damped to still lower velocities. Typically, this capture range is significantly smaller than that for classical molasses, where $v_{c} \cong \Gamma / k$. For polarization rotation, the condition guaranteeing linearity of the damping force is $k v \ll \delta$, where $\delta$ is the difference in light shifts between the sublevels, whereas for ellipticity-gradient cooling the condition is $k v \ll 1 / \tau_{p}$, where $\tau_{p}$ is the optical pumping time. ${ }^{29}$

The decrease of $v_{c}$ as the intensity decreases (or as the detuning increases, since light shifts and optical pumping rates depend on detuning as well) implies that there will be a lower limit to the temperature. Considering first the polarization-rotation case, in the limit of small intensity and large detuning we have

$$
\delta \sim\left(I / I_{0}\right) \Gamma^{2} / \Delta .
$$

In this limit Eq. (36) implies that

$$
M v_{\mathrm{rms}}^{2} \sim \hbar\left(I / I_{0}\right)\left(\Gamma^{2} /|\Delta|\right) .
$$

Combining the inequality $k v \ll \delta$ with relations Eqs. (38) and (39) yields

$$
M v_{\text {rms }} \gg \hbar k .
$$

Thus for polarization-rotation cooling the rms atomic velocity must be much larger than the recoil velocity to have a linear damping force. If the rms velocity approaches the recoil velocity, the nonlinear damping will lead to a nonMaxwell-Boltzmann velocity distribution.

For ellipticity-gradient cooling the condition on $v$ for linearity of damping is $|k v| \ll \Gamma\left(I / I_{0}\right)(\Gamma / 2 \Delta)^{2}$, since the optical pumping rate is proportional to the optical excitation rate. A similar argument leads to

$$
M v_{\text {rms }} \gg \hbar k(2|\Delta| / \Gamma) \text {. }
$$

For linearity of the damping force both inequalities (40) and (41) require that the velocity be much larger than the recoil velocity. At the same time, the expression for the temperature, Eq. (36), allows the thermal velocity to go below the recoil velocity for some intensity and detuning. 
As such a condition is reached, the polarization-gradient force will no longer be linear in $v$ and will be smaller than that given by Eq. (34) or (35). Dalibard and Cohen-Tannoudji have given an explicit form for the nonlinearity of the force with velocity for the case of ellipticity-gradient cooling, finding [Eq. (4.28) of Ref. 29] that

$$
F=\frac{-\alpha v}{1+v^{2} / v_{c}^{2}},
$$

where the critical velocity $v_{c}$ is given by $k v_{c}=1 / 2 \tau_{p}$. Using their expression for the optical pumping time $\tau_{p}$, and assuming that these results are valid for sodium, for $I / I_{0}=1.25$ and $\Delta=-2.73 \Gamma$ we find for $v_{c} \cong 3 \mathrm{~cm} / \mathrm{sec}$, the recoil velocity $v_{R}$. For these conditions, the numerical results of the Stanford group give a $v_{c}$ of roughly $1 \mathrm{~cm} / \mathrm{sec}$. When $v_{c}$ is less than $v_{R}$, a single photon emission or absorption can take the atom's velocity outside the critical velocity. Under such conditions, the effectiveness of the polarization-gradient cooling will be seriously reduced. The polarization-gradient cooling limit for these conditions leads to a rms velocity that is actually higher than $v_{c}$, so it is clear that the velocity distribution will be nonthermal and have an average energy higher than that predicted by the linear damping.

Of course, the intensity at which $v_{c}$ goes below either $v_{R}$ or the cooling-limit velocity will depend on the specific atomic system and the nature of the polarization-gradient cooling being employed. For example, the Stanford group finds that $v_{c}=1 \mathrm{~cm} / \mathrm{sec}$ for ellipticity-gradient cooling, for the same conditions that give $v_{c}=10 \mathrm{~cm} / \mathrm{sec}$ for polarizationrotation cooling. Nevertheless, for any system there will be a combination of intensity and detuning at which $v_{c}$ is less than $v_{R}$ or the cooling limit. At that point, the polarizationgradient force becomes ineffective, and the classical molasses force begins to govern the cooling process. The question of the critical velocity and the laser intensity conditions needed to avoid problems are also discussed in Ref. 29.

The damping coefficients discussed above were calculated in Refs. 28 and 29 by determining the force on an atom constrained to move at constant velocity through the optical field. In fact, an atom does not move in this way but is constantly subject to fluctuations in its velocity owing to the absorption and emission of photons. Ungar et al. have pointed out that the steady-state damping force is reached only after a time comparable with the optical pumping time, during which time the fluctuations in the velocity can be considerable. They have performed Monte Carlo calculations of the atomic motion and obtained force-versus-velocity curves that take into account the hysteresis and averaging that occur. The damping coefficient so obtained is smaller and the critical velocity is higher than given by the steadystate calculation. The change is particularly dramatic for the ellipticity-gradient case, in which the critical velocity is comparable with the recoil velocity. The inclusion of these effects makes the damping for the ellipticity-gradient and the polarization-rotation cases quite similar. For $I / I_{0}=1.25$ and $\Delta=-2.73 \Gamma, \alpha \cong 0.7 \hbar k^{2}$ with a critical velocity of $\sim 10$ $\mathrm{cm} / \mathrm{sec}$. This implies a velocity-damping time of $\sim 5 \mu \mathrm{sec}$.

\section{B. Recoil Limit}

The previous discussions of new laser-cooling mechanisms show that the minimum achievable temperatures may be close to those associated with the recoil energy from a single photon, $E_{R}=\hbar^{2} k^{2} / 2 M$. We expect this energy to set a lower limit on the temperature. Wineland and Itano first examined this problem, assuming Maxwell-Boltzmann velocity distributions. ${ }^{3}$ We reexamine the problem assuming twolevel Doppler cooling, without putting any restrictions on the form of the velocity distributions, and find that the discrete recoil does indeed set a limit corresponding to one recoil energy in each degree of freedom. The theoretical discussions of Sections 1-3.A essentially ignore the discrete nature of laser cooling. When the minimum temperature is comparable with the recoil energy, as in the case of polarization-gradient cooling, a continuous treatment may not be justified. In addition, there are some cases for which laser cooling has been proposed, such as hydrogen and positronium on the Lyman- $\alpha$ transition, for which the recoil energy is so large $\left(\hbar^{2} k^{2} / 2 M \gtrsim \hbar \gamma\right)$ that the effects of a finite recoil cannot be neglected even in the context of Doppler cooling. It is important then to examine the effect of a large recoil on the laser-cooling process, including its effect on detuning, equilibrium temperatures, and velocity distributions.

The approximation that ensures a linear damping force, $|k v| \ll \Gamma$, can be recast in terms of the recoil velocity. Using Eq. (13) and the equipartition theorem, we can replace $\Gamma$ by $2 M v_{\min } 2 / \hbar$, where $v_{\min }$ represents the rms velocity at the Doppler-cooling limit. This yields the condition

$$
k v_{\min } / \Gamma=\hbar k / 2 M v_{\min }=v_{R} / 2 v_{\min } \ll 1,
$$

where $\hbar k / M=v_{R}$ is the recoil velocity. It is apparent that the condition for a linear damping force is equivalent to stating that the recoil velocity is small compared with the smallest rms velocity of the atoms in the molasses. Note that in the limit of large detunings, where, from Eq. (12), $M v^{2}=\hbar|\Delta|$, the condition that $|k v| \ll|\Delta|$ can still be rewritten as $v_{R} \ll v_{\text {rms. }}$. For sodium $v_{R}=3 \mathrm{~cm} / \mathrm{sec}$, while the rms velocity for one-dimensional molasses at the minimum temperature is $30 \mathrm{~cm} / \mathrm{sec}$.

As the condition on the velocities is weakened, the first departure from the simple analysis applied to Eq. (2) is the appearance of terms of higher order in velocity in the damping force and the appearance of a velocity-dependent term in the heating component. This perturbative approach is discussed by Castin et al. ${ }^{46}$ Since we are also interested in the extreme condition in which $k v_{R} \gg \Gamma$, we shall consider a solution to the problem that can be applied for arbitrary linewidths and recoil velocities.

The discrete nature of laser cooling, especially when the recoil velocity is large, is ideally suited for Monte Carlo simulations. We performed these simulations in three dimensions in the low-power limit, with no standing-wave effects included. The emitted radiation pattern was assumed to be isotropic. The linewidth of the laser was always assumed to be negligible compared with $\Gamma$. Velocity and speed distributions were obtained by sampling the velocity of a single particle in molasses, in the spirit of the ergodic theorem. Temperatures were extracted from fits to the velocity distributions. It is important when doing simulations to sample the velocity of the atom at fixed times, not per photon, since when $k v_{R} \gg \Gamma$ an atom can spend vastly different amounts of time between absorptions, depending on its velocity. This will turn out to have a critical effect on recoil-dominated situations.

A test of the Monte Carlo simulation is the case of sodium, 


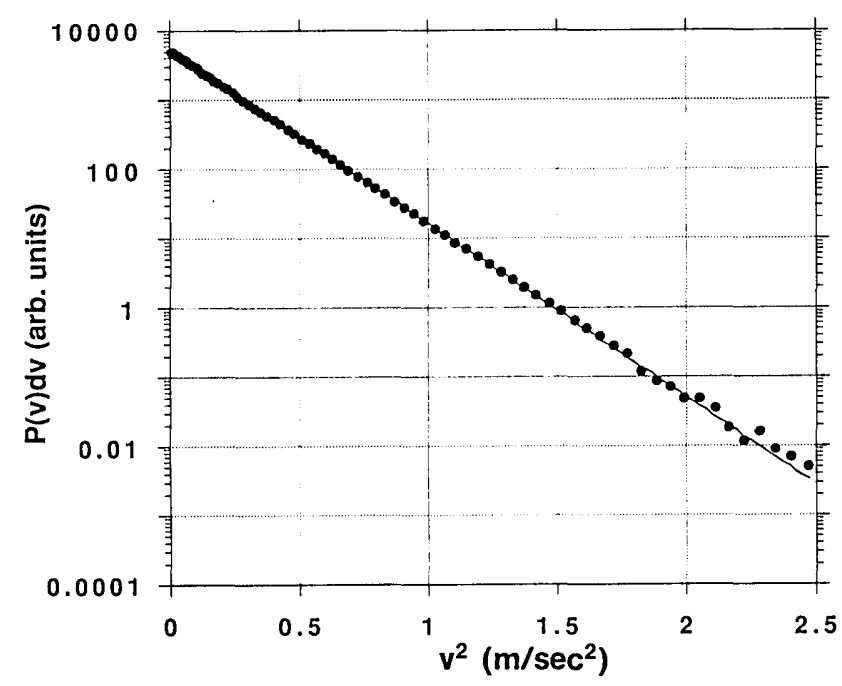

Fig. 3. Velocity distribution from a Monte Carlo simulation of three-dimensional sodium molasses with $\Delta=-\Gamma / 2$ and $I / I_{0} \ll 1$. The straight line represents an exponential fit to the data, giving a temperature of $239 \mu \mathrm{K}$.

for which classical molasses theory predicts a minimum temperature of $238 \mu \mathrm{K}$ at $\Delta=-\Gamma / 2$. To obtain a MaxwellBoltzmann velocity distribution one needs not only a force linear over the range of velocities relevant for cooled atoms but a velocity-independent momentum diffusion constant, as well. This is nearly the case for sodium. Figure 3 is a plot of the velocity distribution from a Monte Carlo simulation of sodium molasses. It is convenient to plot the logarithm of the distribution versus $v^{2}$. A Maxwell-Boltzmann distribution is then a straight line whose slope is inversely proportional to the temperature. A fit to the distribution is displayed, resulting in a temperature of $239 \mu \mathrm{K}$, in good agreement with classical molasses theory. Note also that the distribution of velocities is well described by a MaxwellBoltzmann distribution. As shown by Ref. 46 , the effect of a finite recoil raises the temperature by $E_{R} / 2 k_{B}=1.2 \mu \mathrm{K}$ for sodium at this detuning of $-\Gamma / 2$. This is not resolvable because of the $\sim 3 \%$ statistical uncertainty. Simulations were run for cases with somewhat larger recoils, for which Doppler cooling is still expected to be a good approximation but recoil effects are more prominent. For large detunings, classical molasses theory predicts a temperature that approaches $-\hbar \Delta / 2 k_{B}$. We find that, at large detunings, the finite recoil actually lowers this temperature by $E_{R} / 2 k_{B}$, in agreement with the perturbative treatment of Ref. 46 .

Numerical simulations were run for cases in which the recoil velocity is not small compared with the rms velocity of the cooled atoms. We characterize such systems with a recoil parameter, $\eta=k v_{R} /(\Gamma / 2)=4 E_{R} / \hbar \Gamma$. The simulations reveal two main features of laser cooling in this recoil-dominated $(\eta>1)$ regime. Wineland and Itano ${ }^{3}$ showed with simple energy balance arguments that, when recoil is included, cooling is possible only for a laser detuning of $\Delta<-k v_{R}$, where we define $\Delta=0$ to be the center of the transition for a ground-state atom at zero velocity. ${ }^{47}$ This is to be contrasted with the condition $\Delta<0$ for Doppler cooling, where recoil is neglected. Our Monte Carlo results show that there is a detuning condition that is even more severe, namely, that the detuning must be red of resonance by approximately $\Delta<$
$-2 k v_{R}=\eta \Gamma$ or more. A similar result, obtained by using a different method, is found in Ref. 46. It is difficult to specify the critical detuning condition exactly from the Monte Carlo simulations, because for detunings near this condition the fluctuations in the energy are large, requiring a great deal of computer time to determine whether equilibrium has been reached. Figure 4 shows the rms velocity of an atom with $\eta=10^{4}$ at a detuning of $-2.0 k v_{R}$ as a function of the number of scattered photons. The large fluctuations in the velocity are characteristic of this region near the critical detuning condition. For detunings comfortably satisfying the condition, the atom remains stably cooled without any large velocity excursions.

The reason for the detuning condition, $\Delta<-2 k v_{R}$, and the discrepancy with the condition $\Delta<-k v_{R}$ from Ref. 3 are connected with the velocity distributions. Figure 5 shows a series of velocity distributions for differing values of $\eta$. As in the case of sodium, when the recoil is negligible the distributions look much like a Maxwell-Boltzmann distribution. When the recoil parameter is large, a hole in the velocity distribution is burned at the place where the laser is tuned. This is not too surprising: the recoil due to the absorption and emission of a photon is sufficient to take the atom out of resonance-a velocity-space optical pumping process. Atoms that random walk into the correct velocity range to be

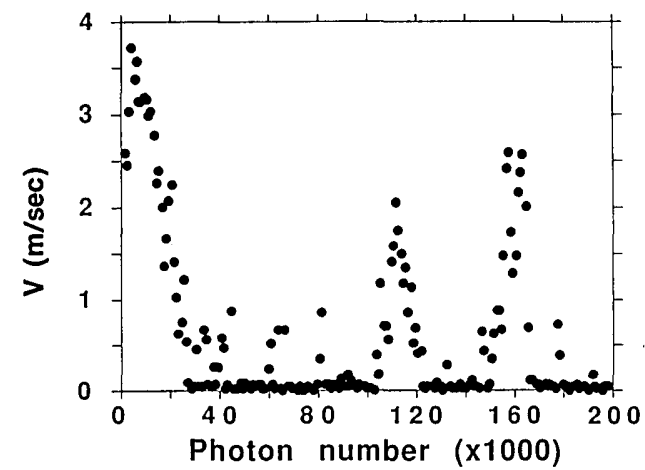

Fig. 4. $V_{\mathrm{rms}}$ versus number of scattered photons for $\eta=10^{4}, \Delta=$ $-\eta \Gamma$, and $v_{R}=3 \mathrm{~cm} / \mathrm{sec}$.

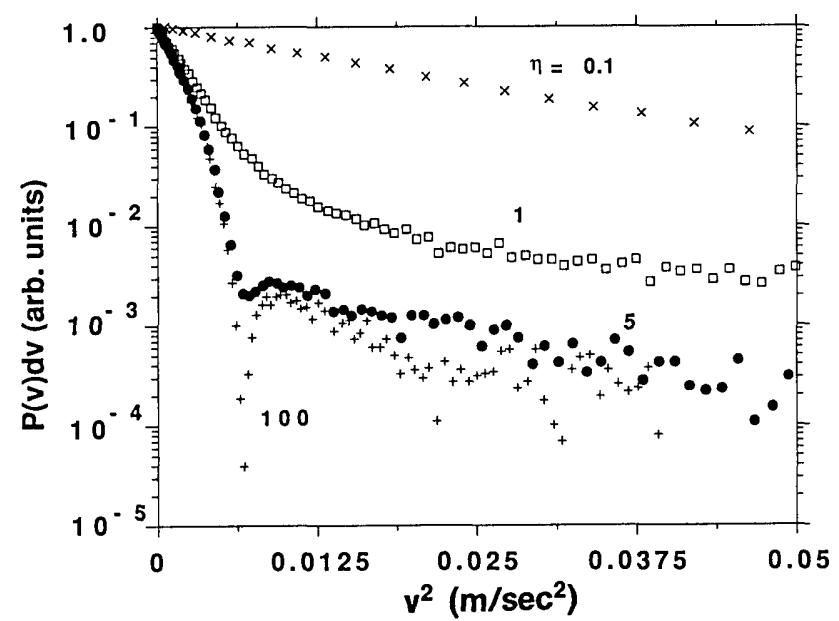

Fig. 5. Velocity distributions for $\eta=0.1,1,5,100$, showing the appearance of a hole for large $\eta$. In all cases $v_{R}=3 \mathrm{~cm} / \mathrm{sec} . \quad \Delta=$ $-1.1 \eta \Gamma$, except for $\eta=0.1$, where $\Delta=-\Gamma / 2$. 


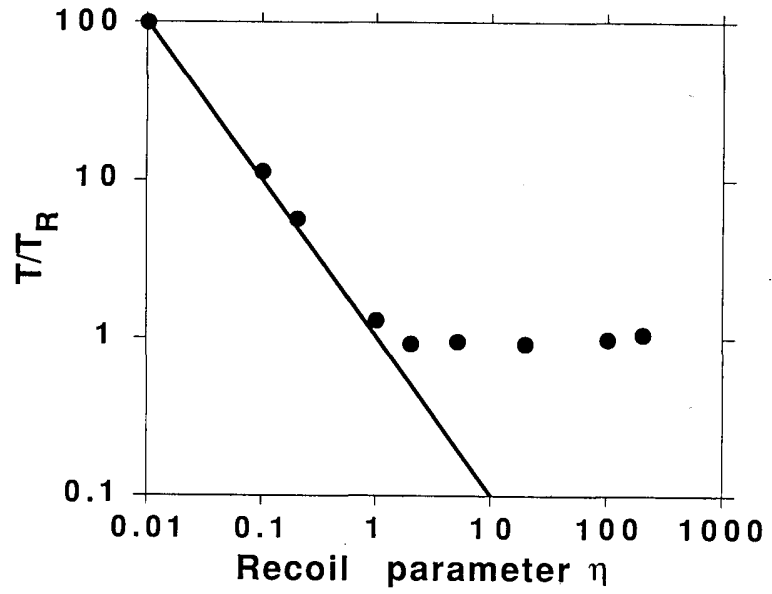

Fig. 6. Minimum temperature versus recoil parameter. Note that the minimum temperature approaches $T_{R}$ for $\eta>1$, and $\hbar \Gamma / 2$ (solid line) for $\eta<1$. For $\eta \gg 1$, the temperature was derived by using only the low-velocity atoms of the distribution.

resonant with the laser rapidly recoil out of resonance. This will tend to create a hole where the excitation is high, leading to nonthermal velocity distributions. The hole is much wider than the linewidth of the transition. Laser cooling of atoms with a large recoil parameter demonstrates the importance of considering the velocity dependence not only of the cooling force but also of the scattering rate that produces the heating. The existence of the hole in the velocity distribution is a direct consequence of the strong dependence of the excitation rate on velocity.

The hole appears to be a rather minor perturbation on the velocity distribution. One needs only a small number of atoms to fill the hole. The hole is difficult to observe in the speed distribution, since the velocities in the three dimensions are essentially independent. It is most apparent when one is measuring the velocity distribution along a laser beam axis. As we shall see, its importance is significant, although its existence is easy to overlook.

We may assign an effective temperature to the distribution by fitting the low-velocity atoms to a Maxwell-Boltzmann distribution. Alternatively, we can assign an effective temperature from the mean square velocity. This yields similar results because the hole is in a velocity region where there are few atoms for a Maxwell-Boltzmann distribution. As is shown in Fig. 6, the minimum temperature achievable for arbitrarily narrow lines (large $\eta$ ) is $k_{B} T / 2=\hbar^{2} k^{2} / 2 M$ (within 5\%), corresponding to one recoil energy in each degree of freedom. We refer to this temperature as $T_{R}$.

Our result that the minimum temperature is $T_{R}$ should be compared with the result obtained by Wineland and Itano. ${ }^{3}$ They examined recoil-dominated laser cooling analytically and found temperatures well below $T_{R}$ for very narrowlinewidth transitions $(\eta>400)$. This discrepancy is due to a different set of assumptions. Wineland and Itano assume that the velocity distribution is always Maxwell-Boltzmann: the atoms effectively rethermalize between every absorption-emission cycle, and a hole never appears. This assumption would be nearly impossible to satisfy for atoms in optical molasses but is more reasonable for species stored in a trap. The rethermalization always supplies some atoms at the velocities that interact strongly with the laser. It ap- pears that the lack of the hole increases the effectiveness of laser cooling, permitting a detuning closer to resonance and a temperature well below $T_{R}$. When the hole is formed, as in our model, the cooling is less effective. The extent of the Lorentzian wings of the excitation line over the Gaussian shape of the velocity distribution determines the outcome. The large breadth of the hole (many linewidths) indicates that the wings of the excitation are quite important. When the detuning $\Delta>-2 k v_{R}$ apparently the wings do more heating than cooling. They extend over many more low- than high-velocity atoms because of the shape of the distribution. The heating of the low-velocity atoms dominates over the cooling of the high-velocity ones, leading to an unbounded increase in the temperature. When $\Delta<-2 k v_{R}$ our results indicate that the excitation of high velocities is favored enough for cooling to occur. Although we have seen that velocity-space optical pumping limits the temperature for recoil-dominated cooling, it is possible to use such optical pumping to populate coherent dark states to achieve a temperature below the recoil limit in one dimension. ${ }^{48}$

A number of groups including our own have proposed and are working on cooling atomic hydrogen by using the $122-\mathrm{nm}$ Lyman- $\alpha$ transition. ${ }^{49,50}$ Although the linewidth for this transition is very broad (100 MHz), hydrogen is so light and the photon is so energetic that $\eta=0.5$. As can be seen from Fig. 5, the effect of recoil is beginning to appear in the velocity distribution and may affect the cooling process. In addition, the added benefits from polarization-gradient cooling mechanisms will be limited, since the recoil temperature is so close to the Doppler-cooling limit. Another atomic transition that would show the effect of recoil is the $1 s 2 s^{3} S_{1}$ $\rightarrow 1 s 3 p^{3} P_{1}$ transition at $389 \mathrm{~nm}$ in helium where $\eta=0.9$.

The laser cooling of positronium has been suggested. ${ }^{51}$ This is a completely recoil-dominated situation owing to the extremely light mass of the atom. For positronium, the recoil parameter $\eta=240$. The lowest obtainable temperature would essentially be the recoil energy of $300 \mathrm{mK}$. Although this temperature is much larger than $\hbar \Gamma / 2$, it would still be of great utility for positronium experiments. Unfortunately, the detuning condition $\Delta<-2 k v_{R}$ allows detuning only to within 240 linewidths, where the transition probability is so low that extremely high laser powers would be required (recall that the lifetime of ortho-positronium is only $140 \mathrm{nsec}$ ). It might be possible to use a broadband laser, which effectively increases the transition width, but this would also require much more power to drive the transition. It is still possible to decelerate a beam of positronium, but the velocity spread will be increased substantially because of heating if the laser is detuned less than 240 linewidths, which would almost certainly be necessary to obtain reasonable absorption rates.

\section{Pinball}

Atoms in three-dimensional optical molasses move in a complicated standing-wave field having both polarization and intensity gradients. We have considered the damping and heating effects of this field but not the possibility that atoms might be trapped or channeled or undergo scattering from the static dipole-force potentials created by the standing waves. Atoms have been confined and channeled by dipole forces. ${ }^{42,52-57}$ Cold atoms, with energies of the same order of magnitude as the size of the modulations in the dipole poten- 
tials, can scatter, possibly quite strongly, off these modulations. Since the intensity modulations in a standing-wave light field occur on a scale of $\lambda / 2(1 / 4 \mu \mathrm{m})$, the motion of atoms in molasses, which would otherwise have an $\sim 20-\mu \mathrm{m}$ mean free path in the case discussed in Subsection 2.E, may be dramatically altered.

We use a two- or three-dimensional generalization of the two-level theory of Gordon and Ashkin ${ }^{8}$ to study the effects of such potential scattering. This approach, applicable only to a two-level system, takes proper account of all locally varying quantities such as the dipole potential forces, the diffusion coefficient (position-dependent heating), and the velocity-dependent damping forces. We have investigated this system with a detailed Monte Carlo simulation and give here a qualitative description of our results. The depth of the potential wells for $\Delta=-\Gamma$ and $I=I_{0}$ is $\sim 50 \mu \mathrm{K}$ for sodium. When the atoms' mean kinetic energy is several times the depth of these wells, the wells have little effect on the atomic motion. Even the random fluctuations in the atoms' velocity seldom reduce the energy of the atoms to a level at which they are scattered significantly by the potential energy modulations of the light field.

On the other hand, when the atoms have an average kinetic energy equal to or less than the peak-to-valley difference in the local potential energy modulations, their motion will be dominated by scattering effects. These atoms will frequently be trapped inside local potential wells until their energy fluctuations allow them to escape (trapping in threedimensional optical potential wells was considered as early as 1977 by Letokhov et al. ${ }^{6}$ and recently by Kazantsev et $a l .58$. This temporary localization of the atoms in the lattice of a standing-wave potential severely retards their macroscopic diffusive motion. Even when the atoms are not actually trapped inside local potential minima, they can scatter off the lattice of potential wells much like a ball rolling across a board with hills and depressions in it. The ball will scatter off bumps or channel along troughs much as in a game of pinball, seldom moving in a direct path for long. In this situation it is impossible for the ball to build up a large velocity before being scattered. Similarly, an atom in the lattice of standing-wave potential wells in molasses will scatter if its energy is low enough.

The inclusion of potential well scattering in these Monte Carlo simulations of otherwise classical molasses has noticeable, but not large, effects on the temperature and lifetime of, and drift velocities produced in, molasses. Artificially lowering the heating (diffusion) term in the simulations by a factor of 2 , however, greatly increases the scattering effects. The polarization-gradient damping mechanisms discussed above would be expected further to enhance the role played by such scattering processes. Nonetheless, the pinball effect does not seem to be required to explain any of the observed features of molasses, with the possible exception of the lifetime.

\section{EXPERIMENTS WITH OPTICAL MOLASSES}

\section{A. Early Experiments}

The earliest experiments that used symmetric or counterpropagating laser beams to cool atoms were performed by Balykin et al.59 In those experiments a two-dimensional configuration of laser beams was used to cool the transverse

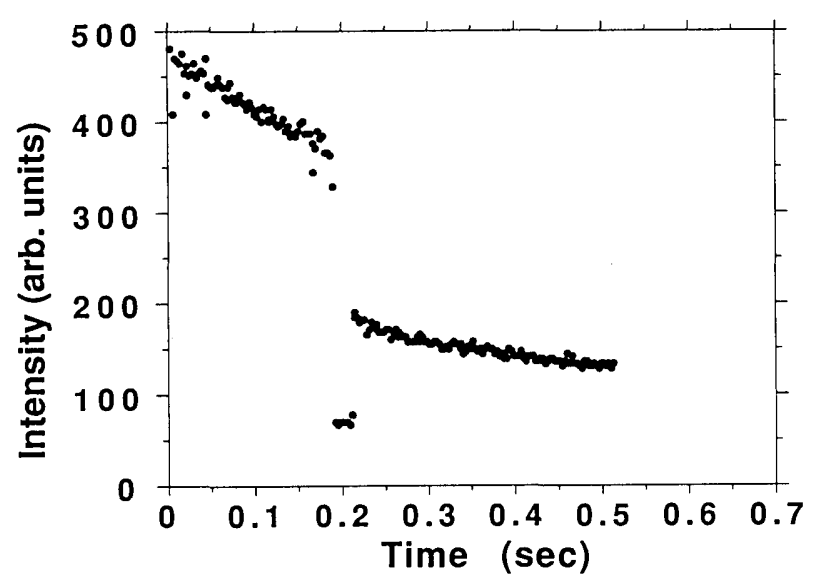

Fig. 7. Fluorescence intensity of the optical molasses versus time in the R\&R method (see text). The molasses beams are turned off for a period $t_{\text {off }}$ lasting, in this case, $20 \mathrm{msec}$. The data are from R\&R measurements taken in our laboratory.

motion of atoms in a sodium atomic beam. By bouncing a collimated light beam off the inside of a reflecting cone, they formed an axisymmetric light field. An atomic beam that traveled along the axis of this cone was thus irradiated uniformly from all directions transverse to its propagation direction. This configuration damped the transverse motion of the atoms, increasing the phase space density and collimating the beam. Their first experiments demonstrated transverse cooling from 40 to $3.5 \mathrm{mK}$ in some tens of microseconds spent in the light field. Later efforts provided transverse cooling from 190 to $17 \mathrm{mK} .{ }^{59}$

The concept of optical molasses as a three-dimensional viscous confinement mechanism (as opposed to simply three-dimensional cooling ${ }^{1}$ ) was proposed and demonstrated by Chu et al. in $1985^{21}$ and independently proposed in Ref. 22 near the same time. In the Bell Labs experiments a pulsed source of sodium atoms was used from which some of the atoms were laser cooled by the frequency-chirp method ${ }^{38}$ to velocities $(\sim 30 \mathrm{~m} / \mathrm{sec})$ capturable by the molasses. The molasses confinement beams were formed by combining two lasers with frequencies separated by approximately the 1.7$\mathrm{GHz}$ sodium ground-state hyperfine splitting to prevent optical pumping (see Subsection 4.B). After the molasses was loaded, it decayed by diffusion of the atoms out of the confinement region. The molasses lifetime was measured by monitoring the decaying fluorescence. Decay times of $\sim 0.1$ sec from a 0.8 -cm-diameter region were observed.

These experiments also introduced the first ballistic temperature measurement technique: The loaded molasses is first allowed to decay for a brief period of time. The molasses laser beams are then blocked for a time $t_{\text {off }}$, of the order of milliseconds. During $t_{\text {off }}$ the atoms move ballistically, and some fraction of them leaves the confinement volume. The laser beams are then unblocked, causing the remaining atoms again to fluoresce. (See Fig. 7.) The ratio of the fluorescence intensity just after the beams are turned back on to the intensity just before they are blocked is a measure of the fraction of atoms that remains in the region during the period of ballistic motion. The plot of this ratio versus $t_{\text {off }}$ is compared with model calculations. If one assumes a Maxwell-Boltzmann initial velocity distribution and an initial 
spatial distribution, a temperature can be extracted from the data. We call this technique release and recapture (R\&R).

Using this R\&R technique, the Bell Labs group found a temperature of $240_{-60}^{+200} \mu \mathrm{K}$ for a detuning that was not well known. This was in good agreement with the minimum temperature predicted by Doppler cooling (see Subsection 2.A.2). Later, the temperature of cesium optical molasses was also measured by the same technique to be $100_{-30}^{+100} \mu \mathrm{K}$, consistent with the Doppler-cooling limit of $120 \mu \mathrm{K}$ for cesium. ${ }^{60}$ These experiments suggested that the classical theory of optical molasses was adequate to describe the experiments and supported the belief that the Doppler-cooling limit was the lowest temperature achievable in such experiments.

Soon, however, experiments showed serious discrepancies with the classical theory of molasses under conditions when one had good reason to believe that the classical theory was valid. Early experiments ${ }^{23}$ in our laboratory measured the molasses brightness and diffusive lifetime as a function of the laser intensity and detuning. A typical experiment involved scanning the molasses laser and simultaneously recording a saturated absorption spectrum as a frequency reference. During the scan, brightness was recorded with a photomultiplier tube. A decay rate experiment was performed as follows: As the laser frequency was slowly scanned the source of cold atoms was chopped on and off, allowing the molasses first to load and then to decay. A typical loading-decay curve is shown in Fig. 8. The figure shows the brightness increasing as the molasses accumulates slow atoms. Once the stopping laser and the atomic beam are chopped off, the molasses decays slowly as the atoms diffuse out of the molasses region.

These measurements revealed startling discrepancies with the classical theory. The first surprise was that the optical molasses was still effective at slowing and accumulating atoms at $\Delta \approx-3 \Gamma$. Indeed, the molasses was brightest at detunings near $-2 \Gamma$ to $-2.5 \Gamma$. The simple classical molasses analysis given in Section 2 indicates that the best molasses (lowest temperatures, largest damping, longest lifetimes, and therefore probably the brightest fluorescence) would be

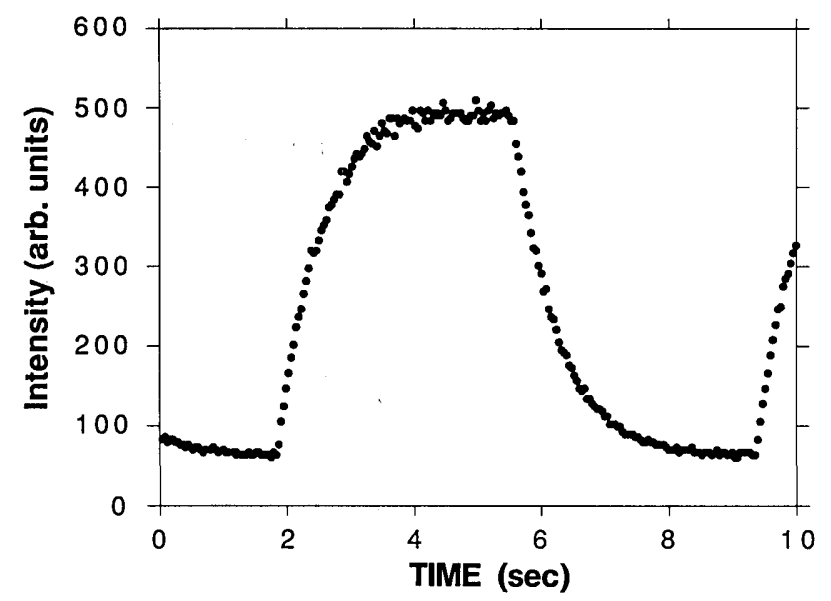

Fig. 8. Molasses fluorescence versus time as the source of slow atoms is chopped on and off. The molasses is allowed to load for $\sim 4$ sec, whereupon the atomic beam and its cooling laser are chopped off, allowing the molasses to decay.

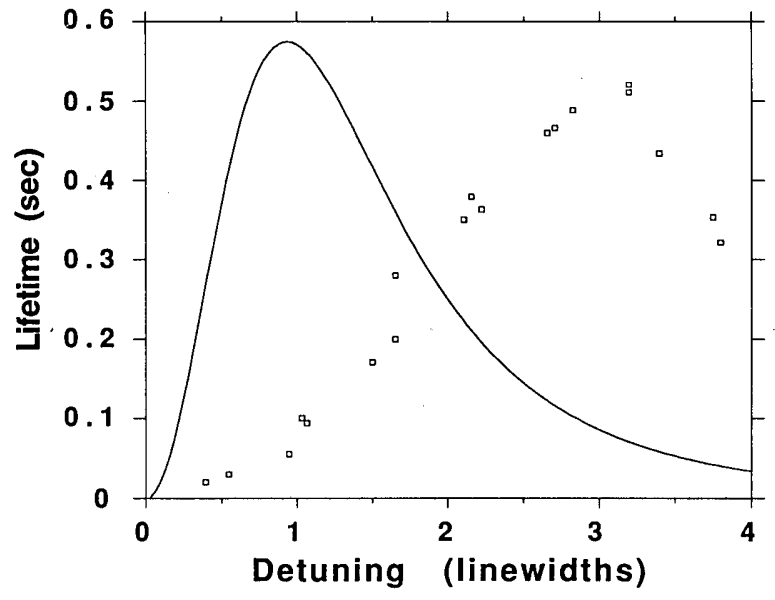

Fig. 9. Molasses lifetime versus laser detuning to the red of resonance. The open boxes are early experimental measurements made in our laboratory, while the solid curve is the prediction of Eq. (30) with $I / I_{0}=0.5$.

expected at detunings near $-0.5 \Gamma$. The theoretical classical molasses lifetime derived in Subsection 2.E is shown in Fig. 9 and compared with our early experimental lifetime measurements. The lifetime $\tau_{M}$ of the molasses peaks at $\Delta \approx-3 \Gamma$. A further surprise was that the lifetime of the molasses was seriously degraded by the application of magnetic fields as small as $50 \mu \mathrm{T}$. This was unexpected since the Zeeman shifts $(0.014 \mathrm{MHz} / \mu \mathrm{T})$ for such a field are small compared with both $\Delta$ and $\Gamma$.

An even clearer discrepancy from the classical theory involves the effect on molasses decay rate of intensity imbalance within a beam pair. The classical theory [see Eq. (33)] predicts that an imbalance of $1 \%$, for an average one-beam intensity of $7 \mathrm{~mW} / \mathrm{cm}^{2}$, leads to a drift velocity of $\sim 4 \mathrm{~cm} / \mathrm{sec}$ and an added decay rate of $10 \mathrm{sec}^{-1}$ for a 4-mm-radius molasses. In fact, we found experimentally that the imbalance had to be $>30 \%$ to produce such a large added decay rate $1 / \tau_{M}$. Figure 10 shows experimental results for the increase in molasses decay rate due to intensity imbalance versus the percentage imbalance. The data were obtained by attenuating one of the retroreflected beams. A theoretical curve showing the classical molasses prediction derived from the calculations of Subsection 2.E is also presented. The drift-induced decay rate is a factor of 10 to 20 times smaller than that predicted. A similar insensitivity to imbalance was reported by Chu et al. for supermolasses. ${ }^{24}$

These early experiments demonstrate that the dependence of molasses lifetime on detuning, magnetic field, and imbalance contradicts the classical theory. As we shall see below, all these features can now be understood, at least qualitatively, in terms of polarization-gradient laser cooling. For example, the two dashed curves shown in Fig. 10 are derived from calculations in Ref. 29 of the polarizationgradient damping coefficients (albeit for different angular momenta) given in Eqs. (34) and (35) and fit the experimental data much better than does the classical theory.

While these experiments indicated difficulties with the classical theory, it was the measurement of the temperature of optical molasses that finally provided convincing evidence of the complete inadequacy of the classical theory to describe optical molasses. 


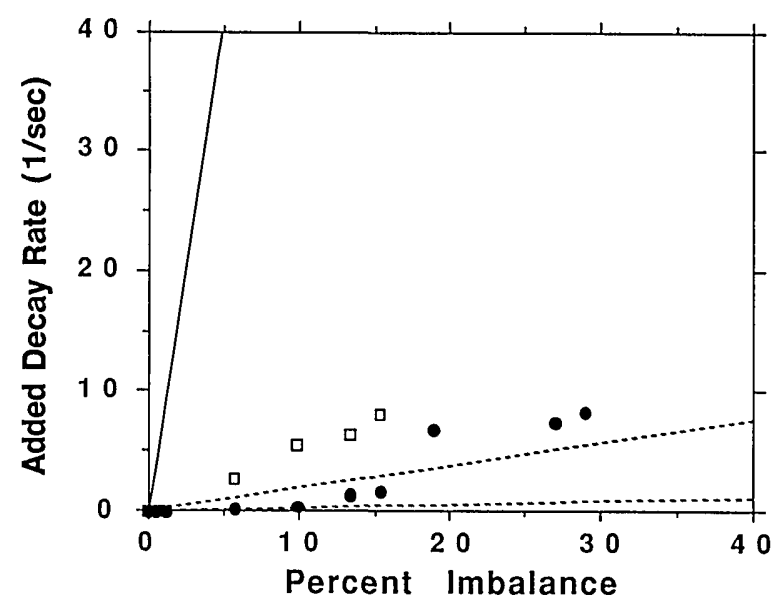

Fig. 10. Added decay rate of the molasses due to imbalance in one pair of beams versus the percent intensity imbalance. The filled circles and open boxes indicate two independent experimental data sets. The solid curve indicates the classical molasses predictions of Subsection 2.E with $\Delta=-2 \Gamma$ and $I / I_{0}=0.5$. The dashed curves are from similar calculations made using the damping coefficients of the polarization-gradient forces of Section 3. The upper dashed curve is from the polarization-rotation damping of Eq. (34), and the lower dashed curve is from the ellipticity-gradient damping of Eq. (35).

In experiments first reported in Ref. 25 we measured the temperature of the atoms released from the molasses, employing a technique different from the R\&R method described above. We added a probe laser beam beneath the molasses, and separated by one to two molasses diameters, and observed the time of flight (TOF) of the released atoms from the molasses region to the probe. ${ }^{61}$ This technique is less sensitive to certain systematic errors associated with the R\&R method. For example, determination of the temperature from $R \& R$ measurements requires knowledge of the spatial distribution of the atoms before they are released. Such geometric considerations become insignificant in the TOF method as the separation between the molasses and the probe is increased. If the viewing volume for recaptured atoms is not sufficiently restricted, the measurements become confused by atoms that fluoresce in a pair of beams outside the molasses. Although the TOF method avoids these problems, it has uncertainties of its own, which are discussed in some detail in Subsection 4.B.3 below.

We measured the temperature by recording the probeinduced fluorescence as a function of the time after the atoms were released from the molasses. The temperature was determined by comparing the peak of the arrival-time distribution with that of the expected distribution calculated by assuming an initial temperature for the atoms. Figure 11 shows a recent example of a TOF measurement with the results of such a calculation for sodium atoms at 25 and 250 $\mu \mathrm{K}$. The measured temperatures here and in Ref. 25 are obviously inconsistent with the Doppler-cooling limit and are consistent with a temperature near $25 \mu \mathrm{K}$. We estimated a systematic error of $\pm 20 \mu \mathrm{K}$ for the TOF method.

To check this surprising result we performed three additional kinds of temperature measurement (including an $R \& R$ measurement in which careful account was taken of the problems listed above), each sensitive to a different set of atomic velocity components. These have been described in Ref. 25. They supported the TOF measurements and ruled out the possibility that the molasses was as hot as the classical Doppler-cooling limit. Later, temperatures below the Doppler limit were also observed at Stanford, ${ }^{27}$ using sodium, and at the Ecole Normale, ${ }^{26}$ using cesium and, in one dimension, metastable helium.

Since the initial measurements of sub-Doppler-limit temperatures, we have made improvements in our experimental technique that have allowed us to refine the conclusions given at that time. We stated in Ref. 25 that, based on our $R \& R$ measurements, the temperature did not change within experimental error when an acousto-optic modulator was used to turn off the molasses beams in less than $1 \mu \mathrm{sec}$ instead of the usual 30- $\mu \mathrm{sec}$ turnoff time of our mechanical shutter. We also observed no dependence of the temperature on the intensity over a limit range. Subsection 4.B below discusses more precise measurements, revealing that both intensity and turnoff time affect the data and that the slow turnoff suppresses the intensity dependence of the temperature.

We also reported ${ }^{25}$ that the temperature exhibited a strong dependence on the magnetic field. At that time the magnetic field had been nulled by optimizing the brightness of the molasses with respect to field. We later found that the temperature is a more sensitive measure of the field. After adjusting the magnetic field to give the lowest temperature, we found that the field that had been used in Ref. 25 was $\sim 50 \mu \mathrm{T}$ different from that which gives the lowest temperature. Measurements with a flux gate magnetometer confirmed that the optimum average field is in fact zero to within $3 \mu \mathrm{T}$. The gradient of any field component is less than $30 \mu \mathrm{T} / \mathrm{cm}$ in any direction, and increasing this gradient by at least a factor of 2 has no measurable effect on the temperature. We had stated, on the basis of Hall probe measurements, that the field was zero to within $20 \mu \mathrm{T}$, so we were apparently in error by more than twice our estimated uncertainty.

\section{B. Recent Experiments}

In this section we shall first give a brief description of our apparatus as it was used in our most recent experiments. We shall discuss general characteristics of the experimental system and details of the laser configuration used in our

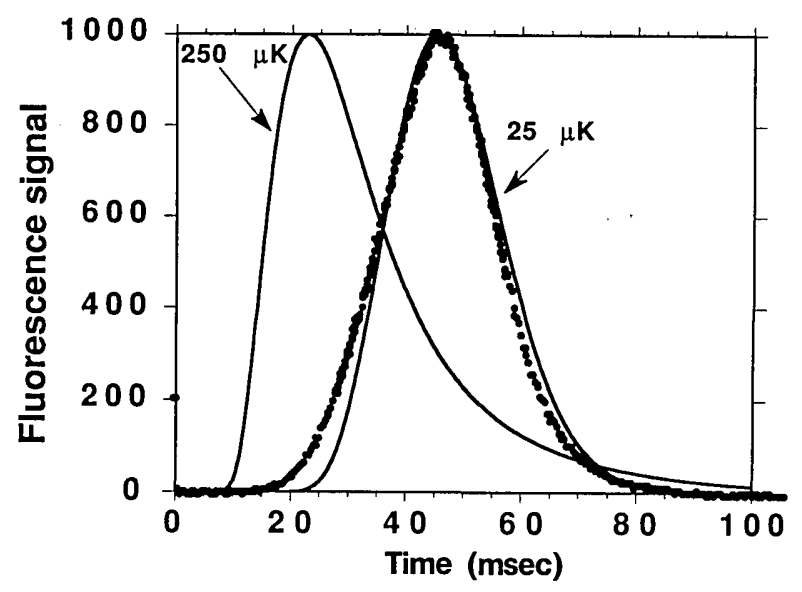

Fig. 11. TOF data and results of numerical calculations at 25 and $250 \mu \mathrm{K}$, all normalized to the same height. $\Delta=-2.5 \Gamma$. The data points show fluorescence intensity versus time, and the solid curves indicate the expected signal. 


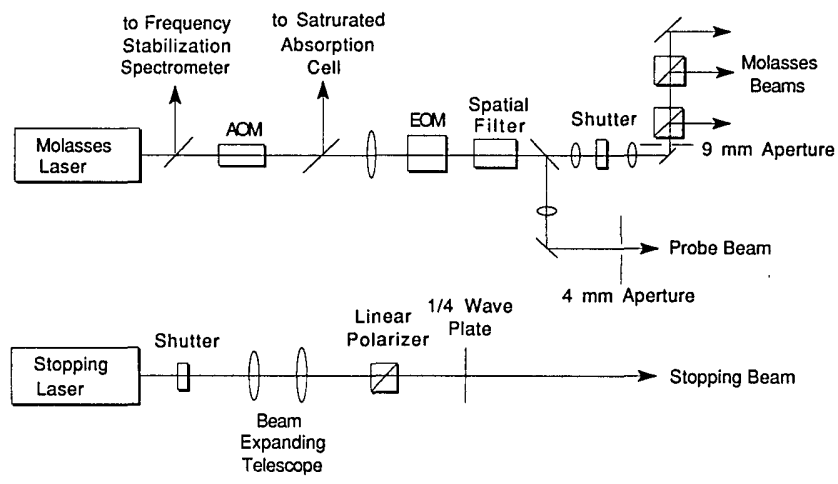

Fig. 12. Block diagram of the optical system. The spatial filter was not present for most of the data. An electro-optic modulator is designated by EOM.

laboratory to produce sodium optical molasses. (See Fig. 12.) Typical values for some experimental parameters are given and, unless otherwise noted, are used in the experiments described in the following sections. We discuss progress that we have made in improving our experimental techniques since the earlier measurements and present more-recent investigations of the properties of optical molasses. In particular, we have studied the dependence of the temperature on a variety of experimental conditions for comparison with the predictions of the polarization-gradient theories summarized in Section 3. We shall see that the majority of the recent data agree, at least qualitatively, with the predictions of the polarization-gradient theories. We also discuss in detail the experimental errors associated with our temperature measurements.

\section{Experimental Configuration}

Our optical molasses is loaded with slow atoms from a sodium atomic beam that propagates along the axis of a Zeemantuning tapered solenoid and is continuously cooled by a counterpropagating laser beam. ${ }^{22}$ Atoms that are not quite stopped on reaching the end of the solenoid leak past the edges of the cooling laser and travel into the experimental region. Measurements of the velocity distribution of these escaping atoms in the molasses region show that the rather broad distribution peaks near $30 \mathrm{~m} / \mathrm{sec}$. The molasses region, which is located $\sim 20 \mathrm{~cm}$ downstream from the end of, and $\sim 2.5 \mathrm{~cm}$ above, the magnet axis (see Fig. 13), intercepts some small percentage of these escaping slow atoms and viscously captures and accumulates them.

The molasses is formed at the intersection of three retroreflected beams propagating along orthogonal axes. The light in each pair of beams is linearly polarized and orthogonal to the polarization of the other two pairs. The beams are roughly Gaussian $\left(1 / e^{2}\right.$ radius $\left.=4 \mathrm{~mm}\right)$ and are apertured to a 9-mm diameter. In the most recent experiments a spatial filter ensures Gaussian beams. A total power less than or equal to $45 \mathrm{~mW}$ is split into three equal beams, each of which is retroreflected to form one of the beam pairs. This maximum value gives a single-beam on-axis intensity of approximately $60 \mathrm{~mW} / \mathrm{cm}^{2}$. The average single-beam intensity within the $1 / e^{2}$ radius of the Gaussian is then $\approx 26 \mathrm{~mW} / \mathrm{cm}^{2}$. Averaging over the sublevels in the $F=2 \rightarrow F^{\prime}=3$ transition gives a saturation intensity $I_{0}=13.5 \mathrm{~mW} / \mathrm{cm}^{2}$. Therefore, for the maximum available power, the average single beam I/
$I_{0} \approx 1.9$, and we typically use approximately a factor of 4 less. We adopt the convention of quoting the measured laser power before the beam is split.

The laser is tuned near the $3 S_{1 / 2}, F=2 \rightarrow 3 P_{3 / 2}, F^{\prime}=3$ transition, and an electro-optic modulator is used to impress $1732-\mathrm{MHz}$ sidebands upon the carrier. Each sideband contains $\sim 10 \%$ of the total power, and the upper sideband is used to prevent optical pumping to the $F=1$ ground state (see Fig. 14). The chosen frequency provides a resonant repumper on the $F=1 \rightarrow F^{\prime}=2$ transition when the carrier is tuned $20 \mathrm{MHz}$ below the $F=2 \rightarrow F^{\prime}=3$ resonance. Adjusting the sideband frequency by small amounts $( \pm 5$ $\mathrm{MHz}$ ) has little effect on the molasses.

The beams are collimated to better than $1 \mathrm{mrad}$, pass through antireflection-coated vacuum windows, and are retroreflected external to the vacuum chamber. Window and mirror losses are $\lesssim 1 \%$, and the absorption losses in the molasses itself, even with the densest molasses obtained, are negligible.

The laser used to produce these beams is optically isolated from the retroreflected beams by an $80 \mathrm{MHz}$ acousto-optic modulator (AOM), which can also serve as a fast shutter. A mechanical shutter at the focus of a beam-expanding telescope operates more slowly but with complete extinction. The laser is locked to an external cavity that is in turn locked to a polarization-stabilized tunable $\mathrm{He}-\mathrm{Ne}$ laser. The satu-

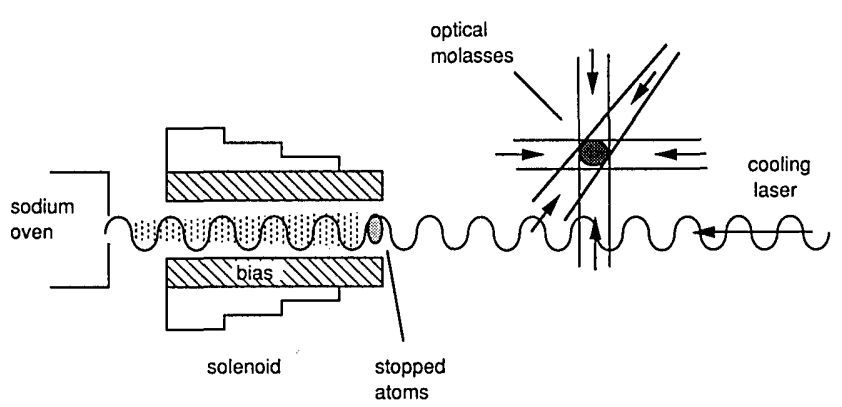

Fig. 13. Sketch of the apparatus, showing the relative position of the Zeeman tuning magnet and the molasses region (not to scale).

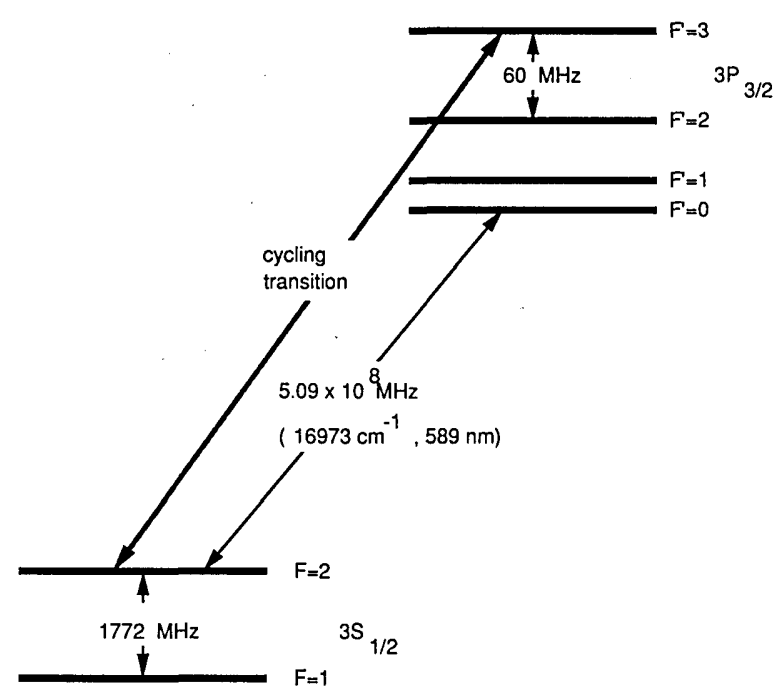

Fig. 14. Sodium energy-level diagram (not to scale) indicating the cycling transition used for laser cooling. 
rated absorption spectrum from an auxiliary vapor cell is continuously monitored and provides an absolute frequency reference near the atomic resonance frequency. Magnetic fields present in the molasses region are nulled, within $\sim 3$ $\mu \mathrm{T}$, by using three pairs of orthogonal coils. The measured base vacuum pressure in the experimental region is less than $10^{-6} \mathrm{~Pa}\left(\sim 10^{-8}\right.$ Torr).

The probe laser is derived from the same laser as the molasses beams and includes the repumping sidebands. It is split off before the mechanical shutter so that it may be on while the molasses beams are off.

\section{Experimental Results}

The new polarization-gradient theories summarized in Subsection 3.A make a number of predictions about the behavior of molasses when experimental parameters such as laser detuning, intensity, and magnetic field are varied. With refinements in our experimental technique we have been able to investigate these predictions in some detail. All the data in this subsection were acquired with the TOF method in a magnetic field nulled by minimizing the temperature. In Subsection 4.B.3 we discuss in detail other systematic errors of the order of $20 \mu \mathrm{K}$ remaining in our absolutetemperature measurements. Day-to-day changes in the geometry often resulted in $10-20-\mu \mathrm{K}$ shifts. However, during a day's run the geometry remains relatively fixed, and we are sensitive to changes in the temperature of a few microkelvins. Therefore we are confident that the dependences of the temperature on the varied parameters are significant.

We begin with a discussion of experiments investigating the effect of the shut-off time of the molasses beams, because it affects the interpretation of our other measurements. We measured the temperature, using an AOM to turn off the beams with a variable ramped turn-off time. This type of experiment was first reported by the Stanford group. ${ }^{2}$ Our data are shown in Fig. 15. The initial molasses power was $13 \mathrm{~mW}$ in the sum of all three beams, and the detuning was approximately $-20 \mathrm{MHz}$. The light intensity was ramped down linearly in a time $t_{r}$, which could be varied. The extinction ratio of the AOM was 700:1 within a few hundred nanoseconds after a fast turnoff. We used a mechanical shutter to extinguish the light completely $20-80$

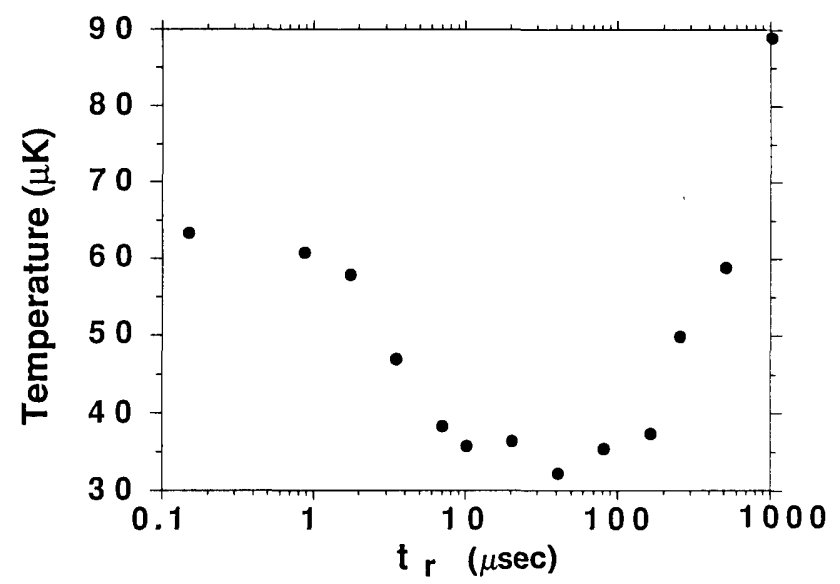

Fig. 15. Temperature measured by TOF as a function of turn-off time $t_{r}$. The quantity $t_{r}$ is the time that it takes to ramp down the molasses laser beam intensity with an AOM. $\mu$ sec after the AOM had switched. The data for a $30-\mu \mathrm{sec}$ AOM ramp agree well with those obtained with a mechanical shutter. The data are similar to the Stanford results, which were obtained by using much higher laser powers, except that our temperatures are lower.

The Stanford group suggested that the turn-off time effect could be due to cooling by adiabatic lowering of dipole potential wells. ${ }^{62}$ They pointed out that, at these low temperatures, the dipole force due to the standing waves in the molasses region could trap some of the atoms. As the lasers are shut off, the receding walls of the dipole wells could cool the atoms by adiabatic expansion if the atomic oscillation period in the wells were small compared with the shut-off time.

We propose a different explanation for the temperature decrease with increasing $t_{r}$ in Fig. 15 . If the light intensity is turned down slowly compared with the molasses equilibration time $\tau_{\text {damp }}$ defined by Eq. (7), then the temperature will reflect that of the molasses during the final $\tau_{\text {damp }}$ of the shutoff. Since the new theory predicts that the temperature varies as the intensity, we expect lower temperatures for longer $t_{r}$. From the figure we deduce that $\tau_{\text {damp }}$ is of the order of $3 \mu \mathrm{sec}$. This is in rough agreement with the analytic and numerical predictions quoted in Subsection 3.A and in good agreement with the Monte Carlo results of Ref. 28 for a velocity range comparable with that implied by our measured temperature. This time is significantly shorter than the equilibration time predicted by the classical theory of Subsection 2.B.

We have confirmed this interpretation in two ways. First, we have observed directly the dependence of the temperature on the light intensity predicted by the polarizationgradient theories. The data are shown in Fig. 16. The squares represent the data obtained with a fast AOM shutoff; circles show the temperature measured by using a mechanical shutter. As predicted by Eqs. (36) and (37), the dependence is linear. The smaller slope of the mechanical shutter data is easily explained if one supposes that for the mechanical shutter data the atoms simply equilibrate with a molasses whose average power is that of the last few microseconds of the shut-off. The slope of the fast shut-off data is $\sim 80 \mu \mathrm{K}$ per $I / I_{0}$ in a single beam. We may compare this, for example, with Eq. (36), which has a slope of $35 \mu \mathrm{K}$ per $I / I_{0}$. We note, however, that a quantitative comparison is difficult because of the one-dimensional nature of the theory.

A second confirmation was an experiment with a slightly modified shut-off procedure from that used to obtain the data in Fig. 15. In Fig. 17a we show schematically the molasses beam intensity as a function of time for this experiment. We used an AOM to turn down the light from the normal power of $13 \mathrm{~mW}$ to $P_{\text {low }}$ in $200 \mathrm{nsec}$, where it remained for $50 \mu \mathrm{sec}$ before being extinguished completely by the AOM. A mechanical shutter followed $10 \mu \mathrm{sec}$ after the AOM shut-off. The temperature was measured as a function of $P_{\text {low. }}$ Figure 17b shows the data. Just as in the AOM data of Fig. 16, the lower temperature of low-power molasses is clearly evident. Because the shut-off time in Fig. 17a is fast, adiabatic cooling cannot account for the low temperatures, nor is it necessary to invoke adiabatic cooling during the shut-off to explain the results of Fig. 15. All this does not rule out the possibility that adiabatic cooling occurs, but it does imply that the equilibration to the temperature asso- 


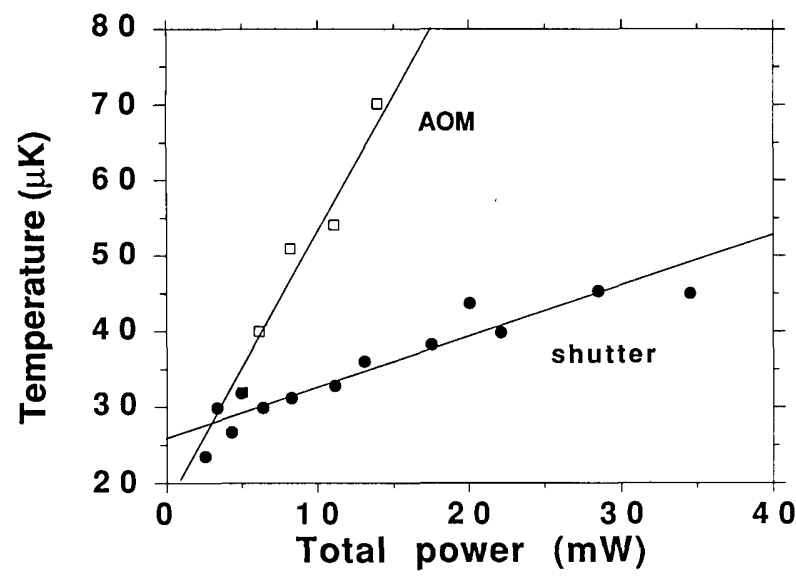

Fig. 16. Temperature measured by TOF versus molasses laser power. The power was measured before the beginning of the shutoff. The AOM shutoff took $200 \mathrm{nsec}$ (squares); the mechanical shutoff took 20-30 $\mu \mathrm{sec}$ (filled circles). The power indicated is the power in the molasses laser beam before it was split into thirds and retroreflected. $\Delta=-2 \Gamma$.

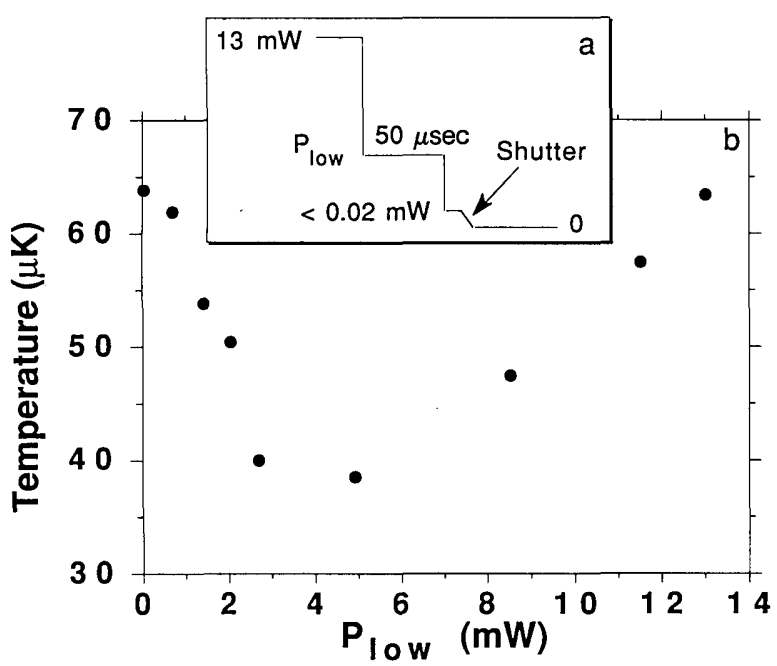

Fig. 17. a, Molasses laser power as a function of time for the data in b. The mechanical shutter shuts off approximately $10 \mu \mathrm{sec}$ after the AOM. b, Temperature versus $P_{\text {LOw }}$ showing that low-power molasses beams cool the atoms despite a fast shut-off. $\Delta=-2 \Gamma$.

ciated with lower intensity is the dominant factor in producing lower temperatures for slow turn-off.

Figure 17b also shows that at powers below $3 \mathrm{~mW}$ (total $I /$ $I_{0}$ of $\left.\sim 1\right)$ the temperature ceases to fall. This supports the prediction of Subsection 3.A [see the discussion following Eq. (42)] that the polarization-gradient force loses effectiveness at low powers as the velocity range over which it acts becomes small. The sharp rise in temperature shown in Fig. 15 at times longer than $100 \mu \mathrm{sec}$ can also be explained by the loss of polarization-gradient cooling. The polarization-gradient cooling disappears well before the end of a $100-\mu \mathrm{sec}$ ramp, so the atoms spend a significant amount of time in low-power laser beams. The classical molasses force should still operate, but its equilibrium temperature is very high, $\sim 500 \mu \mathrm{K}$ at $\Delta=-2 \Gamma$, where these data were taken, so we expect the atoms to be heated.

We have additional evidence for this heating. We have performed an experiment in which the molasses power was turned off slowly (in $\sim 20 \mu \mathrm{sec}$ ), but not completely, with an AOM. The slow turn-off ramps the molasses to a low temperature. After the ramp, a power of $0.14 \mathrm{~mW}, 1 \%$ of the initial power, remained incident upon the molasses region for a variable heating time $t_{h}$. Figure 18a shows the laser intensity versus time for this experiment: Figure $18 \mathrm{~b}$ shows the temperature as a function of $t_{h}$. The data clearly show a linear heating. Using Eq. (10) and assuming that $I / I_{0}=$ 0.01 , we find that the heating rate is of the same order of magnitude as that shown in the data. This experiment also demonstrates that one must be careful when using an AOM as a shutter to make sure that the light is sufficiently extinguished. Following the AOM turn-off with a mechanical shutter, a procedure also reported in Ref. 62 , avoids these heating problems when the extinction of the AOM is insufficient.

The data of Fig. 15 reveal that the true temperature of the atoms while the laser beams are at their steady-state power is best measured by shutting the molasses lasers off in a time much less than $3 \mu \mathrm{sec}$; otherwise the temperature will reflect the laser power during the last several microseconds of the shut-off. In the experiments discussed later in this section the data were taken by using a slow mechanical shutter. Therefore all the temperature measurements correspond to molasses at a much lower intensity than before the start of the shut-off. The dependences that we report are still valid.

The polarization-gradient theory suggests that there is a sensitive dependence of the temperature on magnetic field. (Recall that the classical molasses theory does not predict a large sensitivity to magnetic field). The new theory, however, depends on optical pumping and ac Stark shifts between different states in the $F=2$ ground-state manifold. The presence of a magnetic field can cause Larmor precession and Zeeman shifts of magnetic sublevels that are comparable with the optical pumping rates and ac Stark shifts. One can estimate the field at which the Larmor precession of magnetic sublevels should begin to interfere with optical pumping by observing that the optical pumping time should be of the order of 1-10 $\mu$ sec. The ground-state Zeeman shift and therefore the precession frequency among different hyperfine states is of the order of $10 \mathrm{kHz} / \mu \mathrm{T}$. Thus, at a field

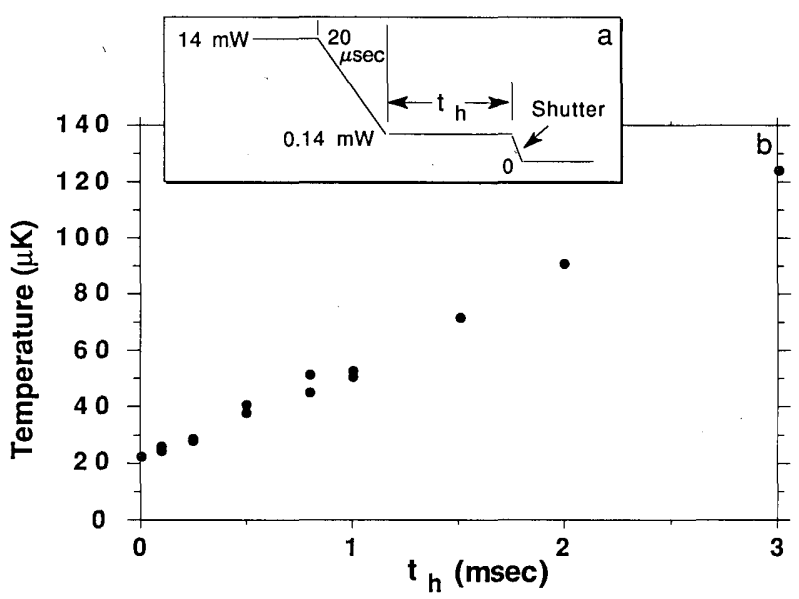

Fig. 18. a, Molasses laser power versus time for the data in Fig. b. $\mathrm{b}$, Temperature versus $t_{h}$, showing rapid heating of the atoms in very-low-power $(0.14-\mathrm{mW})$ molasses. 


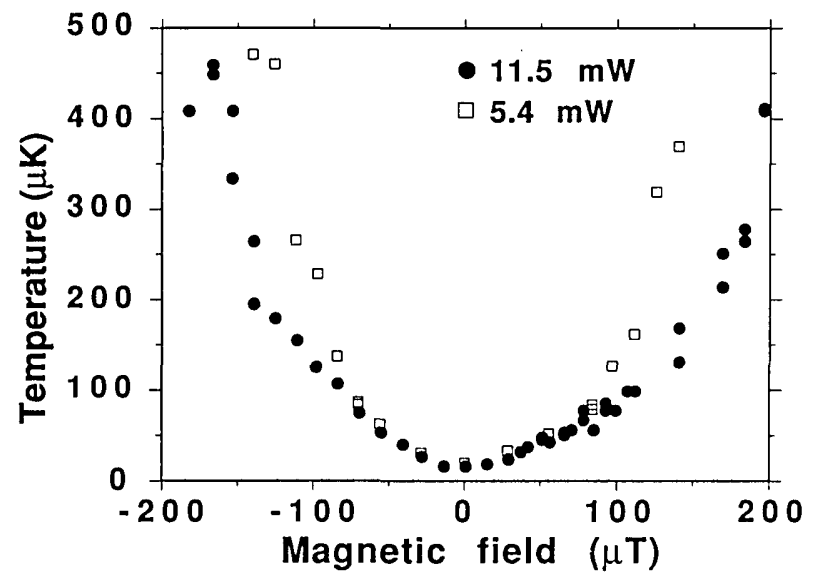

Fig. 19. Temperature versus magnetic field. The data were taken with a mechanical shutter. The two powers displayed were measured before the shutoff. $\Delta=-2 \Gamma$.

of $10-100 \mu \mathrm{T}$, the Larmor precession frequency is of the same order of magnitude as the optical pumping rate. The Larmor precession will compete with the optical pumping and interfere with the polarization-gradient damping. The polarization-gradient theory of laser cooling also suggests that the magnetic field dependence of the temperature will vary with laser intensity. As the intensity is increased, the optical pumping time will decrease. This means that at higher intensity it will require a higher magnetic field to degrade the polarization-gradient force by the same amount. Figure 19, which shows molasses temperature versus magnetic field for two different laser intensities, qualitatively verifies both of these predictions. The effective variation in power was significantly smaller than what is shown in the figure because mechanical shutters were used.

The new theories of laser cooling all depend on the existence of a polarization gradient in the radiation field. Hence if this gradient is reduced we expect the temperature to rise. We have examined the dependence of the temperature on the polarization of the molasses laser beams. Under normal conditions, the (linear) polarization is such that the polarization of each pair of beams is orthogonal to the other two pairs. When the polarization of one of the beam pairs is rotated parallel to the polarization of one of the other pairs, the degree of polarization gradient is smaller. We have rotated the polarization of each pair of molasses beams. We find that when the polarization is rotated from $0^{\circ}$ to $90^{\circ}$, the temperature increases monotonically from 25 to $\sim 50 \mu \mathrm{K}$. Behavior similar to this has also been observed by the authors of Ref. 34 .

We have measured the dependence of the temperature on the laser detuning $\Delta$. Figure 20 shows data taken with the TOF method. Shown also is the prediction of the classical molasses theory, Eq. (12). The shape of the curve is radically different from that of classical molasses. The most striking feature illustrated by Fig. 20 is that the temperature continues to decrease when the detuning is increased far beyond the classical minimum of $\Delta=-\Gamma / 2$. In the classical theory of molasses the increase in temperature at large detuning comes about because the cooling rate, proportional to $\alpha$, is decreasing as $\Delta^{-3}$, while the heating rate, proportional to $\mathscr{D}_{p}$, is decreasing only as $\Delta^{-2}$. In the polarization-gradi- ent theories of laser cooling the situation is quite different. Both the ellipticity-gradient and the polarization-rotation cases produce temperatures that decrease with increasing detuning, as do the data, although the detailed dependences are different [see Eqs. (36) and (37)]. Our three-dimensional molasses with three orthogonally polarized standing waves will in general have a mixture of ellipticity gradient and polarization rotation, with an additional intensity modulation from the standing waves, preventing a direct quantitative comparison with theory. The increase in temperature at detunings larger than $35 \mathrm{MHz}$ shown in Fig. 20 is probably due to the fact that the $F^{\prime}=2$ level is only $60 \mathrm{MHz}$ to the red of the $F^{\prime}=3$ level. At these large detunings the laser is more nearly resonant with, and to the blue of, the lower transition.

Finally, we report measurements of the molasses decay rate as a function of both magnetic field and laser power. Recall from Subsection 2.E that, if one assumes that the motion of the atoms is diffusive, then the molasses decay rate $1 / \tau_{M}$ is proportional to $D_{p} / \alpha^{2}$. The decay rate versus magnetic field is shown in Fig. 21. The behavior of $1 / \tau_{M}$ is similar to that of the temperature, shown in Fig. 19.

Figure 22 shows the dependence of the decay rate on laser power. In polarization-gradient cooling, $\mathscr{D}_{p}$ is proportional to the laser intensity, while $\alpha$ is independent of it. Thus, if the decay rate is governed purely by polarization-gradient cooling, one expects the decay rate to increase as the intensity increases. The data show quite the opposite. It is interesting to note that the decay rate decreases with power in a manner similar to the prediction of the classical molasses theory. Because of the low temperature and high damping, note that the diffusion lifetime of a molasses governed by polarization-gradient cooling is many seconds. The longest $\tau_{M}$ that we have observed is only $625 \mathrm{msec}$. We have experimentally verified that this lifetime was not limited by our vacuum. Perhaps the decay of the molasses is determined by the probability that an atom's velocity is outside the range over which polarization-gradient cooling acts. If an atom's velocity is outside that range, its motion will be governed by classical molasses. The classical molasses will tend to damp its velocity to a point where the polarization-gradient force is effective again. However, during this time the atom also has an increased probability of escaping the mo-

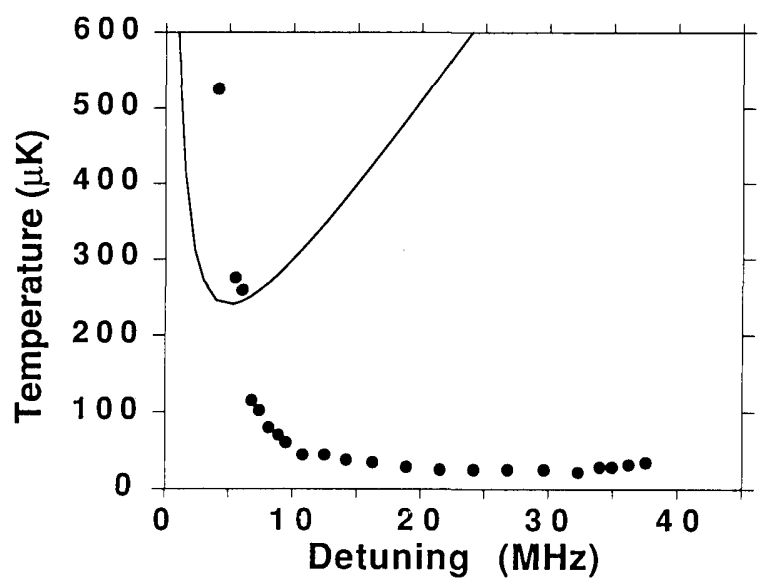

Fig. 20. Measured temperature versus laser detuning $\Delta$, to the red of resonance. Also shown is the prediction of classical, low-power, one-dimensional molasses (solid curve), Eq. (12). 


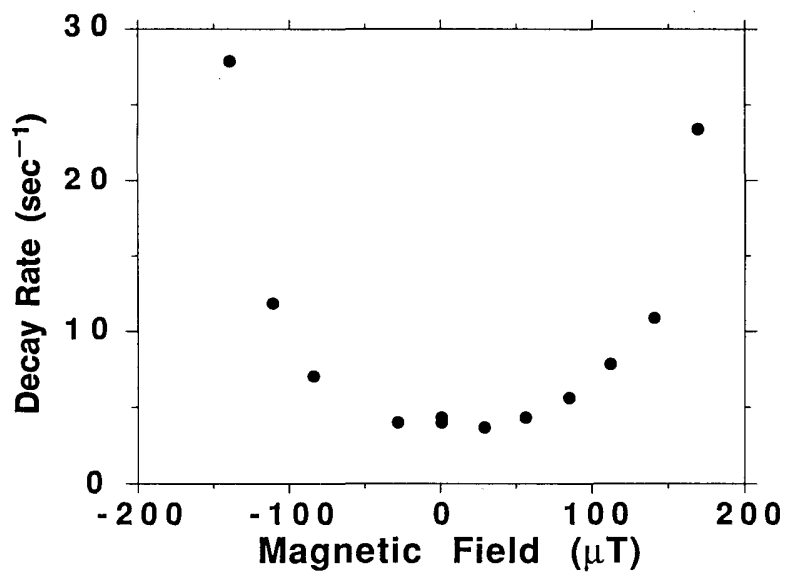

Fig. 21. Molasses decay rate versus magnetic field. $\Delta=-2.5 \Gamma$; power, $10 \mathrm{~mW}$.

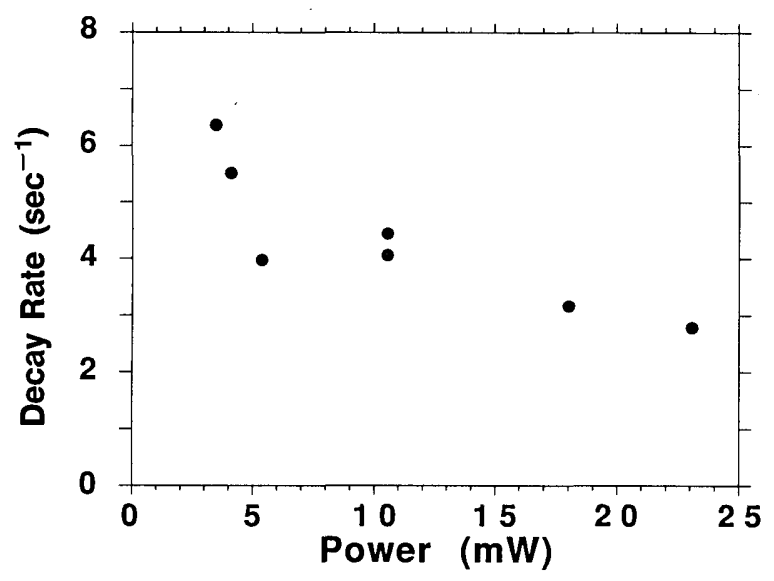

Fig. 22. Molasses decay rate versus laser power. $\Delta=-2.5 \Gamma$.

lasses, since the damping force of classical molasses is much smaller. Both the likelihood of an atom's velocity being outside of the polarization gradient range and the escape probability from the classical molasses are higher at lower intensity. This could explain the behavior observed in Fig. 22. The Stanford group has reported observations of bimodal velocity distributions, ${ }^{34}$ which may be due to similar effects. We note that, if we had a bimodal distribution, with well-separated temperatures such as those quoted in Ref. 34, the presence of the hot peak would have essentially no effect on the determination of our low temperature.

\section{Data Analysis}

The uncertainties in the temperatures quoted above are largely systematic in nature; the short-term repeatability of our data is a small fraction of the total uncertainty. TOF data taken on the same day under the same conditions result in a peak arrival time scatter of $\sim 0.5 \mathrm{msec}$, implying a $1 \sigma$ statistical uncertainty of $\sim 3 \mu \mathrm{K}$.

The systematic uncertainties, on the other hand, are much larger and are due mainly to uncertainties in the distance traveled by the atoms as they fall to and through the probe. To get a feel for the difficulty in making an absolute-temperature measurement, consider that the gravitational potential energy difference (mgh) between atoms at the top and the bottom of a $0.9-\mathrm{cm}$-diameter molasses corresponds to an energy difference $\left(k_{B} T / 2\right)$ equivalent to $\sim 500 \mu \mathrm{K}$. This is much larger than the tens-of-microkelvins kinetic energy that the atoms have when they are first released from the molasses. In this section we shall describe the way in which a temperature is extracted from the experimental data and then discuss individual contributions to the total uncertainty.

To determine the temperature, the data are compared with the results of a numerical calculation of the expected signal. Input includes the relative positions of the molasses and the probe, the molasses and the probe diameters, the length of the probe being imaged by the detection optics, and an experimentally determined estimate of the spatial distribution of atoms in the molasses. For each volume element in a spherically bounded molasses, the program assumes a Maxwell-Boltzmann velocity distribution corresponding to a given temperature and calculates the number of atoms falling, under the influence of gravity, to the probe as a function of time. Integrating over the molasses volume gives an arrival-time distribution corresponding to a TOF signal. Examples of the calculations are shown in Fig. 11 for temperatures of 25 and $250 \mu \mathrm{K}$.

To analyze the TOF data, we compare the experimentally determined peak arrival time with peak arrival times calculated for different temperatures. Other quantities, such as the centroid and the FWHM, also vary with temperature and may be used to interpret the data, although they are generally less sensitive. It is possible, under suitable limits of temperature and/or molasses-probe separation, to derive analytic expressions for the TOF distribution if we assume a point molasses and a point probe. Numerical calculations using these initial conditions are in excellent agreement with the analytic results.

We now discuss the individual contributions to the temperature uncertainty. In general, these are found by varying the parameter of interest in the numerical calculation and observing its effect on the arrival-time distribution.

The main source of geometric error is the measurement of molasses-probe separation. The vertical distance between the centers of the probe beam and the horizontal molasses beam can be measured to $0.5 \mathrm{~mm}$. For temperatures below $\sim 100 \mu \mathrm{K}$, this leads to an uncertainty of $\sim 6 \mu \mathrm{K}$ for a typical center-to-center separation of $1.25 \mathrm{~cm}$. Error due to horizontal displacement of the geometric centers is negligible, as are uncertainties in the size of the molasses. Uncertainties due to the probe will be handled separately.

The uncertainty associated with the distribution of atoms in the molasses is closely connected to uncertainties in determining the molasses-probe separation. Obviously, if all the atoms were at the top of the molasses, the peak arrival time would increase dramatically, and we would assign much too low a temperature. From video images of the molasses we can obtain the distribution of excited-state atoms projected onto a plane. Given the single view that we have of the molasses and the non-Gaussian nature of our molasses laser beams, we can only estimate the position-dependent atomic density. We place the centroid of the distribution at the geometric center of the molasses and assign an uncertainty of $0.5 \mathrm{~mm}$ to this estimate. This translates into a temperature uncertainty of $6 \mu \mathrm{K}$.

The detection process in the probe is the source of most of 
the systematic error in these experiments. Recall that the probe is configured to produce one-dimensional optical molasses. As such, it quickly damps any atomic velocity along the direction of the laser beams. Unlike in the strictly onedimensional problem of Subsection 2.A, atoms in the probe emit photons into all three dimensions. Photons emitted perpendicular to the laser beam produce the same heating as in Eq. (9) but without a counteracting damping force. This causes the velocity distribution perpendicular to the probe to spread as the atoms fall through the probe. Under typical conditions of probe intensity, detuning, and molasses-probe separation, the velocity change is large enough to expel the atoms before they fall completely through the probe. This affects our assignment of a temperature since it changes the effective separation between the molasses and probe. In addition, it becomes more important as the velocity at the probe decreases.

In addition, there is a second, geometric source of probe error. During the course of the data taking, there were dayto-day changes in the probe's profile. While the aperture defining the probe remained a constant $4 \mathrm{~mm}$, the intensity profile across that aperture varied from being fairly uniform to having a high-intensity hot spot a few millimeters in diameter. Uncontrolled changes in the probe's intensity profile across the aperture could shift the effective molasses-probe separation by $\pm 1 \mathrm{~mm}$. We assign $12 \mu \mathrm{K}$ to this systematic uncertainty.

We have performed a detailed Monte Carlo simulation to address the probe detection process. ${ }^{63}$ Here we discuss the results of that simulation for typical probe parameters: power $3 \mathrm{~mW}$, beam diameter $4 \mathrm{~mm}$, laser detuning $-2.5 \mathrm{\Gamma}$, and a Gaussian profile with $1 / e^{2}$ radius $=0.15 \mathrm{~cm}$ centered on the aperture. Under these conditions, an atom falling from the center of a $40-\mu \mathrm{K}$ molasses to a probe centered 1.25 $\mathrm{cm}$ away spends roughly $4 \mathrm{msec}$ inside the probe and scatters $\sim 3000$ photons before being expelled. In addition, most of the photons are scattered within the top $1.5 \mathrm{~mm}$ of the probe, and the atom rarely falls even halfway through the probe. This has been confirmed experimentally: blocking the bottom half of the probe at this power did not change the size or the shape of the TOF signal.

If the power in the probe is reduced to $0.7 \mathrm{~mW}$, the behavior is radically different. An atom is now no longer scattered out of the probe. Instead, it spends $\sim 6 \mathrm{msec}$ in the probe (compared with an 8-msec ballistic transit time) and scatters $\sim 1000$ photons, most of those within $\sim 1 \mathrm{~mm}$ of the probe center. This has also been verified experimentally: reducing the probe power from 0.7 to $0.2 \mathrm{~mW}$ changed the peak arrival time by only $0.5 \mathrm{msec}$, a number comparable with the statistical scatter.

In the experiments discussed in Subsection 4.C. 3 the power and profile of the probe were not well controlled. We assign an error of $12 \mu \mathrm{K}$ to these uncertainties in addition to the uncertainty involving the placement of the probe within the aperture. Combining all uncertainties in quadrature, we arrive at a total uncertainty of $20 \mu \mathrm{K}$ for the TOF data presented in this paper. We note that this error is roughly independent of temperature and therefore gives quite large relative uncertainties at the lowest temperatures.

The numerical calculations have also allowed us to place limits on other possible sources of error. We find that magnetic forces due to field gradients twice those measured at the molasses have no effect on the shape of the TOF curve. In addition, the TOF method is rather insensitive to large radial changes in the molasses density profile.

Finally, we have run our numerical calculations for a variety of different assumptions about the velocity distribution of atoms in the molasses. We looked at the behavior of vertical temperature gradients, radial temperature gradients, and the effect of having different temperatures in the vertical direction and in the horizontal plane. We also examined the possibility of molasses having a homogeneous, isotropic, nonthermal velocity distribution, for example, a mixture of temperatures or a flat velocity distribution having a sharp cutoff. In each situation, the results are consistent with the following simple analysis: As the average vertical velocity of the molasses increases, the peak of the distribution shifts to shorter times. As the average horizontal velocity increases, the atoms spread as they fall to the probe, so fewer atoms are detected. Atoms with a long fall time, such as those with a small vertical velocity or those traveling upward, suffer more horizontal displacement and miss the probe. The result is that the TOF method is most sensitive to atoms near the bottom of the molasses and to those atoms with the lowest horizontal velocities.

We now consider the consequences of this differential sensitivity to velocities when the molasses does not have a Maxwell-Boltzmann velocity distribution. Figure 23 shows the time integral of the TOF distribution as a function of temperature, assuming a point molasses, a point probe, and a molasses-probe separation of $1.25 \mathrm{~cm}$. As the temperature of the molasses rises, the total number of atoms being detected by the probe falls rapidly. If the molasses were composed of equal amounts of atoms at $10,20,30$, and $40 \mu \mathrm{K}$, the temperature seen by the TOF method would look much like a pure temperature of $10 \mu \mathrm{K}$. Similarly, a distribution with equal amounts of 20 - and $100-\mu \mathrm{K}$ atoms would again closely resemble a pure temperature of $20 \mu \mathrm{K}$. In general, for isotropic, homogeneous mixtures of temperatures, the TOF method sees mainly the lowest temperature, while, for two widely separated temperatures, it produces a twopeaked distribution. When considering bimodal TOF distributions, such as presented in Ref. 34 , it is necessary to be aware of this differential sensitivity.

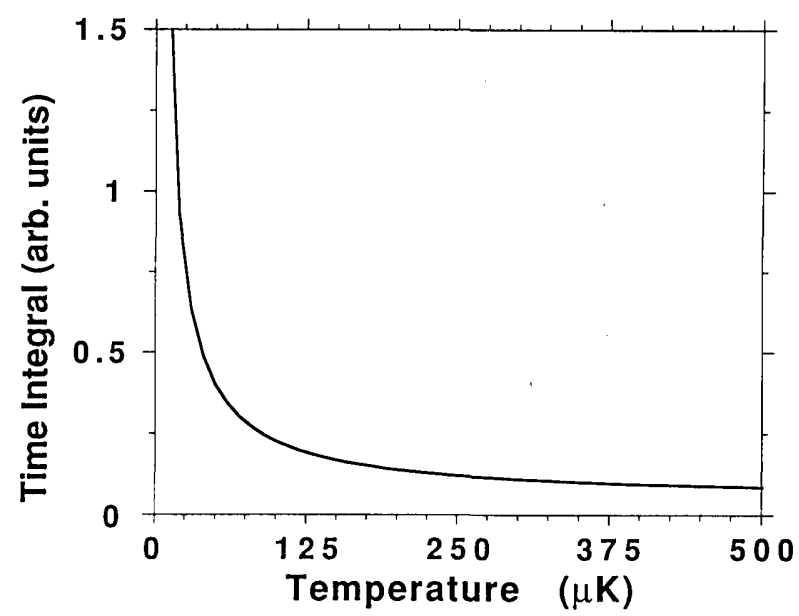

Fig. 23. Time integral of the fluorescence intensity versus molasses temperature showing the differential sensitivity to velocity of the TOF method. 


\section{Improved Geometry}

The systematic error limiting the temperature determination in Subsection 4.B is the shape of the probe and uncertainties about exactly where in the probe the atoms were being detected. This error could be largely eliminated if the probe had a well-defined intensity profile and moderately low power. Atoms falling through such a probe would fluoresce mainly near the center and would be perturbed only slightly. Under these conditions, the detection process can be modeled and included in an improved temperature determination. Furthermore, the molasses beams used in the above experiments were decidedly non-Gaussian. Therefore, to improve the uniformity and symmetry of the molasses and probe beams, the distribution of atoms in the molasses, and the modeling of the detection process, we added a spatial filter. The resulting molasses beams, although roughly $30 \%$ lower in power, now have a smooth Gaussian profile with a $1 / e^{2}$ radius of $5 \mathrm{~mm}$. Similarly, the probe beam is also a well-defined Gaussian with a $1 / e^{2}$ radius of 2.5 $\mathrm{mm}$.

The lifetime of the molasses when the spatially filtered beams are used is now much longer. Lifetimes, as measured at the 1/e point, before spatial filtering were $\sim 300 \mathrm{msec}$ in the best of conditions. After filtering, the lifetime is now $625 \mathrm{msec}$, and there is a corresponding increase in molasses brightness. These results are quoted for a molasses detuned $2.5 \Gamma$ to the red of resonance and a total power in the molasses beams of $10 \mathrm{~mW}$. Also, we are not vacuum limited at a measured pressure of $7 \times 10^{-7} \mathrm{~Pa}\left(5 \times 10^{-9} \mathrm{Torr}\right)$. We note that other groups have used spatially filtered beams for producing optical molasses and have obtained improved performance with them compared with unfiltered beams. $26,27,52,64$

Using this improved molasses and the well-defined probe, we have made new TOF measurements. An example is shown in Fig. 11 for a total molasses laser beam power of 10 $\mathrm{mW}$, a molasses-probe separation of $1.25 \mathrm{~cm}$, a laser detuning $2.5 \Gamma$ to the red of resonance, and a probe power of 0.6 $\mathrm{mW}$. Also shown are the results of the numerical calculation for the best fit of $25 \mu \mathrm{K}$. The results of the Monte Carlo probe simulations have been included in this calculation. After completing a full analysis, we expect our error to be greatly reduced from $20 \mu \mathrm{K}$.

In addition, we have begun exploring the velocity distribution of the atoms in the molasses. Our previous temperature measurements have assumed a well defined MaxwellBoltzmann velocity distribution characterized by a single temperature, and most of the data are in reasonable agreement with that assumption. However, given the differential sensitivity of the TOF method, high-velocity atoms might be present in the molasses but not efficiently detected. Also, the polarization-gradient forces that currently best explain molasses work only over a limited velocity range. Presumably, outside this range, classical molasses is still effective, and the resulting velocity distribution is expected to be nonMaxwell-Boltzmann. The exact nature of this distribution supplies important information about the polarization-gradient forces. Therefore determining the velocity distribution of atoms in the molasses is an interesting test of the new theories.

The shower method ${ }^{25}$ is well suited to this task. Recall that the shower method measures the time integral of the
TOF distribution versus horizontal displacement of the probe and is sensitive to the atoms' horizontal velocity. Since there are no significant horizontal forces in these experiments, the horizontal velocity does not change as the atoms fall to the probe, and the shower method permits a more straightforward determination of the initial velocity profile. In addition, if the molasses is far away, the effect of the atomic spatial distribution in the molasses is minimized, and the technique is less sensitive to all the geometric errors discussed in Subsection 4.B.3. Finally, the shower method has less differential sensitivity to velocity than the TOF method.

We have made recent improvements in the shower method originally described in Ref. 25 . The probe is now located $\sim 6 \mathrm{~cm}$ below the molasses. At this position, the vertical velocity of the atoms at the probe no longer depends much on the atoms' initial vertical velocity or position in the molasses. In addition, a better optical system has been devised to eliminate the uncontrolled variations present in the earlier experiment.

We have obtained preliminary results with this new arrangement, and, indeed, the quality of the data is much improved. Temperatures as derived from measurements taken with the TOF method at the standard $1.25-\mathrm{cm}$ molasses-probe separation and at the new 6-cm separation agree with each other. In addition, both temperatures agree with that obtained from the shower method taken with small to moderate horizontal displacements. Shower data taken with large horizontal displacements show evidence that the velocity distribution of atoms in the molasses is not a pure Maxwell-Boltzmann. However, much work needs to be done in interpreting these results, and a full discussion of these new experiments will be forthcoming.

\section{CONCLUSIONS}

The past year has witnessed a sudden and dramatic increase in our knowledge of the processes involved in laser cooling. The theories of Cohen-Tannoudji and Dalibard and of Chu et al. describe cooling mechanisms entirely unanticipated before the discovery of sub-Doppler temperatures. These mechanisms are much more effective in producing viscous confinement and low mean energies than is Doppler cooling alone. The data of Section 4 show that there is a considerable body of evidence supporting these new theories of laser cooling. Our measurements of temperature versus intensity, detuning, magnetic field, and polarization are all in qualitative agreement with the predictions of polarization-gradient theories, as are measurements of decay rate versus beam imbalance. We emphasize that one must be cautious in making quantitative comparisons because the theories are one dimensional and the analytic expressions apply to transitions different from the one used in sodium. In addition, there are differences in the definition of $I_{0}$ among the Stanford group, the Ecole Normale group, and our group, and it is unclear how to include the effects of the other beams in three dimensions.

A number of problems remain unresolved. Several theoretical predictions, as well as some experimental evidence, indicate that there may be non-Maxwell-Boltzmann velocity distributions in optical molasses. Detailed quantitative predictions of these velocity distributions have yet to be 
made, and more research needs to be done on measurements of the true velocity distribution. Perhaps a better understanding of the velocity distribution together with the inclusion of the pinball effects will allow us to explain the behavior of the power dependence of the decay rate as well as the peculiar properties of supermolasses.

A detailed theoretical explanation of how the magnetic field affects the molasses temperature is also needed. Being able to describe the role played by Larmor precession and Zeeman shifts may allow us to use the magnetic field measurements as a sensitive probe of the polarization-gradient dynamics. It may also help us to distinguish experimentally among various laser-cooling mechanisms.

A full treatment of the polarization-gradient force problem is needed for the $J=2 \rightarrow J^{\prime}=3$ system in three dimensions. This would permit quantitative comparisons with the data in Section 4. Other groups are at work on or have proposed cooling other atomic species. They offer new systems to test polarization gradient theories. It is possible to choose an atomic system in which recoil effects are either much smaller or much larger than those in sodium. This permits experimental investigation of polarization-gradient cooling either unobscured by the effects of recoil or stressing the role of recoil in the cooling process. Atoms with a different hyperfine structure will also permit the study of polarization-gradient cooling at detunings $\Delta<-3 \Gamma$ without the complications presented by the $F=2 \rightarrow F^{\prime}=2$ transition in sodium. Finally, experiments that involve cooling in fewer than three dimensions will greatly simplify the theoretical interpretation of the data since the complicated nature of a three-dimensional standing wave can be avoided. Such experiments are being performed at the Ecole Normale, Stanford, and Stony Brook. ${ }^{26,45}$

We believe that three-dimensional optical molasses will become an important tool for future research in atomic physics. As an example, consider the high-resolution spectroscopic technique of the atomic fountain in which atoms interact twice, once while traveling up and a second time while falling down, with an oscillatory field (optical or rf) in a variation of the Ramsey technique. ${ }^{65}$ We propose using the molasses to launch the atoms collectively so that they have an upward velocity of $\sim 1 \mathrm{~m} / \mathrm{sec}$. This will be done by moving the lower molasses mirror upward at this velocity and then rapidly shutting off the molasses laser beams. Owing to the rapid equilibration time in molasses, all the atoms will follow the motion of the mirror, remaining at rest with respect to the translating standing-wave pattern, and this will produce a group of ultracold atoms with a uniform upward drift velocity. This launch avoids the severe heating problems that would be associated with an upward force produced with a single laser beam. Figure 24 shows a numerical simulation of the number of atoms passing through a plane 2 $\mathrm{cm}$ above the molasses as a function of time after a $1-\mathrm{m} / \mathrm{sec}$ drift velocity has been imparted to a $30-\mu \mathrm{K}$ molasses. The later peak, which is from the atoms as they fall back down, is broadened owing to the thermal velocity distribution within the molasses. Note that this well-resolved second peak will be visible for atoms only with the low temperatures now achievable in optical molasses. The extremely long interaction time ( $\sim 200 \mathrm{msec})$ should be of great benefit for veryhigh-resolution spectroscopy.

As a final comment, we note that most theoretical treat-

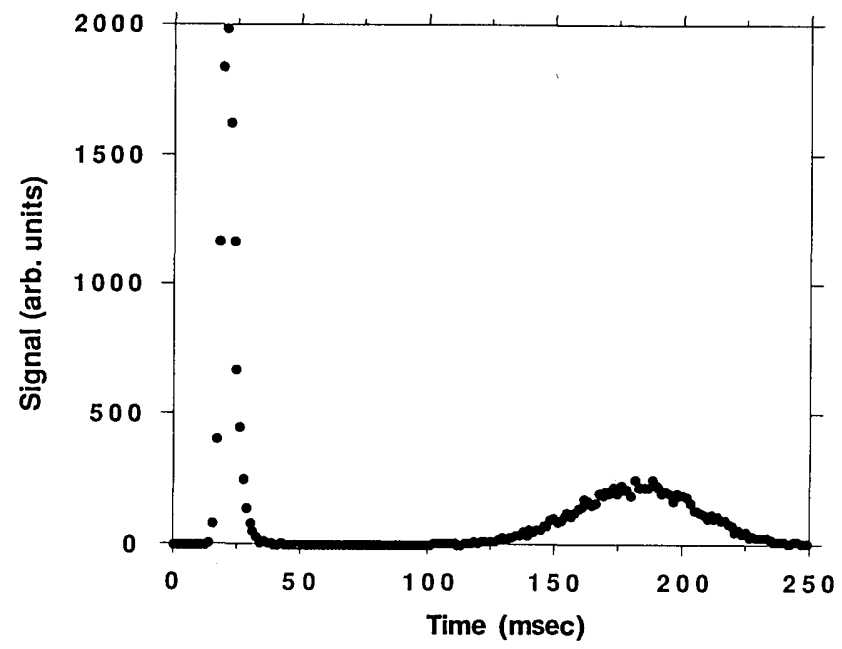

Fig. 24. Fluorescence intensity from a probe versus time after a collective launch of $1 \mathrm{~m} / \mathrm{sec}$ has been given to a $30-\mu \mathrm{K}$ sample of molasses $2 \mathrm{~cm}$ below the probe.

ments of laser cooling to date are based on the assumption that the position and momentum coordinates of the atom can be treated classically (the de Broglie wavelength $\lambda_{B} \ll$ $\lambda)$. This is clearly not the case when the temperature of the atoms approaches the recoil limit where $\lambda_{B}=\lambda$. Furthermore, in the case of sodium molasses, typical dipole potential wells are $\sim 50 \mu \mathrm{K}$ deep, while $T_{R}=2.4 \mu \mathrm{K}$. Semiclassically, the atom would clearly be trapped most of the time if $T$ $\cong T_{R}$. The length scale of the standing-wave potential wells is $\lambda / 2$, smaller than the de Broglie wavelength of the atom. Quantum mechanically, such a trapped atom would have a momentum spread larger than its thermal momentum. This problem with the semiclassical treatment is already relevant since, at the present $20-\mu \mathrm{K}$ temperatures obtained in sodium optical molasses, $\lambda_{B} \approx \lambda / 3$. A fully quantummechanical method for treating some cases of laser cooling in this limit is outlined by Dalibard and Cohen-Tannoudji ${ }^{29}$ and also discussed by Castin et al. ${ }^{46}$

\section{ACKNOWLEDGMENTS}

This research was partially supported by the U.S. Office of Naval Research. In addition, C. E. Tanner and R. N. Watts acknowledge the support of National Research Council-National Institute of Standards and Technology postdoctoral fellowships. We acknowledge the help of our collaborators, Hal Metcalf and Phil Gould. We thank Ed Williams, Steve Chu, Dave Wineland, Wayne Itano, Alain Aspect, and Alan Migdall for helpful discussions. We are especially grateful to Jean Dalibard and Claude Cohen-Tannoudji for communicating their results before publication. In addition, we thank Tom Lucatorto for critically reading this manuscript. Finally, we thank Barry Taylor for his enthusiastic support of our laser-cooling efforts.

\section{REFERENCES AND NOTES}

1. T. Hänsch and A. Schawlow, Opt. Commun. 13, 68 (1975).

2. D. Wineland and H. Dehmelt, Bull. Am. Phys. Soc. 20, 637 (1975).

3. D. Wineland and W. Itano, Phys. Rev. A 20, 1521 (1979).

4. W. Itano and D. Wineland, Phys. Rev. A 25, 35 (1982). 
5. E. Purcell, Harvard University, Cambridge, Massachusetts 02138 (personal communication), cited in Ref. 3.

6. V. Letokhov, V. Minogin and B. Pavlik, Sov. Phys. JETP 45, 698 (1977).

7. W. Neuhauser, M. Hohenstatt, P. Toschek, and H. Dehmelt, Phys. Rev. Lett. 41, 233 (1978).

8. J. Gordon and A. Ashkin, Phys. Rev. A 21, 1606 (1980).

9. R. Cook, Phys. Rev. A 22, 1078 (1980).

10. D. Wineland, R. Drullinger, and F. Walls, Phys. Rev. Lett. 40, 1639 (1978).

11. S. Stenholm, Rev. Mod. Phys. 58, 699 (1986).

12. A. Ashkin, Science 210, 1081 (1980).

13. V. Letokhov and V. Minogin, Phys. Rep. 73, 1 (1981).

14. Prog. Quantum Electron. 8(3 \& 4) (1984).

15. J. Opt. Soc. Am. B 2(11) (1985).

16. D. Wineland, W. Itano, J. Bergquist, and J. Bollinger, eds., Trapped Ions and Laser Cooling, Natl. Bur. Stand. (U.S.) Tech Note 1086 (U.S. Government Printing Office, Washington, D.C., 1985); Trapped Ions and Laser Cooling II, Natl. Inst. Stand. Technol. Technical Note 1324 (U.S. Government Printing Office, Washington, D.C., 1988).

17. W. Phillips and H. Metcalf, Sci. Am. 256, 50 (1987).

18. W. Phillips, P. Gould, and P. Lett, Science 239, 877 (1988).

19. V. Minogin and V. Letokhov, Laser Light Pressure on Atoms (Gordon \& Breach, New York, 1987).

20. A. P. Kazantsev, Phys. Rep. 129, 75 (1985).

21. S. Chu, L. Hollberg, J. Bjorkholm, A. Cable, and A. Ashkin, Phys. Rev. Lett. 55, 48 (1985).

22. W. Phillips, J. Prodan, and H. Metcalf, J. Opt. Soc. Am. B 2 , 1751 (1985).

23. P. Gould, P. Lett, and W. Phillips, in Laser Spectroscopy VIII, W. Persson and S. Svanberg, eds. (Springer-Verlag, Berlin, 1987), p. 64.

24. S. Chu, M. Prentiss, A. Cable, and J. Bjorkholm, in Laser Spectroscopy VIII, W. Persson and S. Svanberg, eds. (SpringerVerlag, Berlin, 1987), p. 58.

25. P. Lett, R. Watts, C. Westbrook, W. Phillips, P. Gould, and H. Metcalf, Phys. Rev. Lett. 61, 169 (1988).

26. J. Dalibard, C. Salomon, A. Aspect, E. Arimondo, R. Kaiser, N. Vansteenkiste, and C. Cohen-Tannoudji, in Atomic Physics 11, S. Haroche, J. C. Gay, and G. Grynberg, eds. (World Scientific, Singapore, 1989), p. 199, and personal communications.

27. S. Chu, Y. Shevy, D. Weiss and P. Ungar, in Atomic Physics 11, S. Haroche, J. C. Gay, and G. Grynberg, eds. (World Scientific Singapore, 1989), p. 636.

28. D. S. Weiss, E. Riis, Y. Shevy, and P. J. Ungar, and S. Chu, J. Opt. Soc. Am. B 6, 2072 (1989).

29. J. Dalibard and C. Cohen-Tannoudji, J. Opt. Soc. Am. B 6, 2023 (1989).

30. J. Dalibard and C. Cohen-Tannoudji, J. Opt. Soc. Am. B 2, 1707 (1985).

31. V. Minogin and O. Serimaa, Opt. Commun. 30, 373 (1979).

32. A. Ashkin, Phys. Rev. Lett. 40, 729 (1978).

33. F. Reif, Fundamentals of Statistical and Thermal Physics (McGraw-Hill, New York, 1965).

34. Y. Shevy, D. Weiss, P. Ungar, and S. Chu, Phys. Rev. Lett. 62, 1118 (1989).

35. One could imagine a two-level atom achieved with a $J=0 \rightarrow J=$ 1 transition with a magnetic field applied to split the $J=1$ levels so much that only one transition, say the $0 \rightarrow 0$ transition, was in resonance with the laser. Two counterpropagating waves, polarized at $\pm 45^{\circ}$ to the magnetic field, would both drive the twolevel transition, but they would create no standing wave in the usual sense of an intensity modulation. There would be, however, a periodic spatial variation in the coupling between the atom and the field since the local polarization from the superposition of the two waves would vary in space even though the intensity of the light would not. This variation would have the same kind of effect as would a true standing wave.

36. J. Dalibard, Thèse de doctorat d'étatès Sciences Physique (Université de Paris, Paris, 1986).

37. J. Javanainen, M. Kaivola, U. Nielsen, O. Poulsen, and E. Riis, J. Opt. Soc. Am. B 2, 1768 (1985);
38. W. Ertmer, R. Blatt, J. Hall, and M. Zhu, Phys. Rev. Lett. 54, 996 (1985).

39. C. Salomon and J. Dalibard, C. R. Acad. Sci. Paris 306, 1319 (1988).

40. H. Wallis and W. Ertmer, J. Phys. B 21, 2999 (1988).

41. J. Dalibard, S. Reynaud, and C. Cohen-Tannoudji, J. Phys. B 17, 4577 (1984).

42. A. Aspect, J. Dalibard, A. Heidmann, C. Salomon, and C. Cohen-Tannoudji, Phys. Rev. Lett. 57, 1688 (1986).

43. R. Feynman, R. Leighton, and M. Sands, The Feynman Lectures on Physics (Addison-Wesley, Reading, Mass., 1963), Vol. I.

44. See, for example, E. Buchwald, Ann. Phys. 66, 1 (1921).

45. J. Dalibard, Laboratoire de Spectroscopie Hertzienne de l'Ecole Normale Supérieure 24 rue Lhomond, F-75231 Paris Cedex 05, France (personal communication); D. Weiss, E. Riis, Y. Shevy, P. Ungar, and S. Chu, J. Opt. Soc. Am. B 6, 2072 (1989); H. Metcalf, Department of Physics, State University of New York at Stony Brook, Stony Brook, New York 11794 (personal communication)

46. Y. Castin, H. Wallis, and J. Dalibard, J. Opt. Soc. Am. 6, 2046 (1989).

47. We set $\hbar \omega_{0}$ equal to the internal energy plus one recoil energy. Wineland and Itano ${ }^{3}$ use a different convention: our $\omega_{0}$ is equivalent to their $\omega_{0}^{\prime}$.

48. A. Aspect, E. Arimondo, R. Kaiser, N. Vansteenkiste, and C. Cohen-Tannoudji, Phys. Rev. Lett. 61, 826 (1988).

49. P. Lett, P. Gould, and W. Phillips, Hyperfine Interact. 44, 335 (1988).

50. T. Hijmans, O. Luiten, I. Setija, and J. Walraven, in Spin Polarized Quantum Systems, S. Stringari, ed. (World Scientific, Singapore, 1989), p. 275.

51. E. Liang and C. Dermer, Opt. Commun. 65, 419 (1988).

52. S. Chu, J. Bjorkholm, A. Ashkin, and A. Cable, Phys. Rev. Lett. 57, 314 (1986)

53. C. Salomon, J. Dalibard, A. Aspect, H. Metcalf, and C. CohenTannoudji, Phys. Rev. Lett. 59, 1659 (1987).

54. P. Gould, P. Lett, P. Julienne, W. Phillips, H. Thorsheim, and J. Weiner, Phys. Rev. Lett. 60, 788 (1988).

55. C. Tanner, B. Masterson, and C. Wieman, Opt. Lett. 13, 357 (1988).

56. V. Balykin, V. Letokhov, Yu. Ovchinnikov, A. Sidorov, and S. Shul'ga, Opt. Lett. 13, 958 (1988).

57. A. Anderson, M. Boshier, S. Harocke, E. Hinds, W. Jhe, and D. Meschede, in Atomic Physics 11, S. Haroche, J. C. Gay, and G. Grynberg, eds. (World Scientific, Singapore, 1989), p. 626.

58. A. P. Kazantsev, G. Surdutovich, and V. Yakovlev, Opt. Commun. 68, 103 (1988).

59. V. Balykin, V. Letokhov, and A. Sidorov, Pis'ma Zh. Eksp. Teor. Fiz. 40, 251 (1984) [JETP Lett. 40, 1026 (1984)]; V. Balykin, V. Letokhov, V. Minogin, Yu. Rozhdestvenskii, and A. Sidorov, Zh. Eksp. Teor. Fiz. 90, 871 (1986) [Sov. Phys. JETP 63, 508 (1986)].

60. D. Sesko, C. Fan, and C. Wieman, J. Opt. Soc. Am. B 5, 1225 (1988).

61. This technique was suggested to us by $\mathrm{H}$. Metcalf.

62. Y. Shevy, D. Weiss and S. Chu, in Spin Polarized Quantum Systems, S. Stringari, ed. (World Scientific, Singapore, 1989), p. 287.

63. The analysis presented in the next two paragraphs was not used in assigning TOF temperatures in Ref. 25. Instead, we assigned an uncertainty based on our then limited knowledge of the probe effect.

64. S. Chu, Department of Physics, Stanford University, Stanford, California 94305 (personal communication); J. Dalibard, Laboratoire de Spectroscopie Hertzienne de l'Ecole Normale Supérieure, 24 rue Lhomond, F-75231 Paris Cedex 05, France (personal communication).

65. A. DeMarchi, Metrologia 18, 103 (1982); R. Beausoleil and T. Hänsch, Phys. Rev. A 33, 1661 (1986). Such an experiment was recently performed by the Stanford group [M. Kasevich, E. Riis, S. Chu, and R. DeVoe, Phys. Rev. Lett. 63, 612 (1989)], using atoms from a Zeeman-assisted radiation-pressure trap but without the launch mechanism that we suggest here. 